OPEN ACCESS

Edited by:

John Michael Streicher

University of Arizona, United States

Reviewed by:

Peter Grace,

University of Texas MD Anderson

Cancer Center, United States

Wei Lei,

Presbyterian College, United States

Michael D. Burton,

The University of Texas at Dallas,

United States

*Correspondence:

Kyle M. Baumbauer

kbaumbauer@kumc.edu

${ }^{\dagger}$ Present address:

Erin E. Young,

Department of Anesthesiology and

Department of Anatomy and Cell

Biology, University of Kansas Medical

Center, Kansas City, KS,

United States

Kyle M. Baumbauer,

Department of Anatomy and Cell

Biology and Department of

Anesthesiology, University of Kansas

Medical Center, Kansas City, KS,

United States

Received: 01 June 2019

Accepted: 30 August 2019

Published: 20 September 2019

Citation:

Knight BE, Kozlowski N, Havelin J,

King T, Crocker SJ, Young EE

and Baumbauer KM (2019) TIMP-1

Attenuates the Development

of Inflammatory Pain Through

MMP-Dependent

and Receptor-Mediated Cell Signaling

Mechanisms.

Front. Mol. Neurosci. 12:220.

doi: 10.3389/fnmol.2019.00220

\section{TIMP-1 Attenuates the Development} of Inflammatory Pain Through MMP-Dependent and

\section{Receptor-Mediated Cell Signaling Mechanisms}

\author{
Brittany E. Knight ${ }^{1}$, Nathan Kozlowski2, Joshua Havelin ${ }^{3,4}$, Tamara King ${ }^{3,4,5}$, \\ Stephen J. Crocker ${ }^{1,6}$, Erin E. Young ${ }^{2,6,7,8 t}$ and Kyle M. Baumbauer ${ }^{1,2,6,7,9 * t}$
}

${ }^{1}$ Department of Neuroscience, UConn Health, Farmington, CT, United States, ${ }^{2}$ School of Nursing, University of Connecticut, Storrs, CT, United States, ${ }^{3}$ Center for Excellence in the Neurosciences, University of New England, Biddeford, ME, United States, ${ }^{4}$ Graduate School of Biomedical Science and Engineering, University of Maine, Orono, ME, United States, ${ }^{5}$ College of Osteopathic Medicine, University of New England, Biddeford, ME, United States, ${ }^{6}$ Institute for Systems Genomics, UConn Health, Farmington, CT, United States, ${ }^{7}$ The Center for Advancement in Managing Pain, School of Nursing, University of Connecticut, Storrs, CT, United States, ${ }^{8}$ Genetics and Genome Sciences, UConn Health, Farmington, CT, United States, ${ }^{9}$ Rita Allen Foundation, Princeton, NJ, United States

Unresolved inflammation is a significant predictor for developing chronic pain, and targeting the mechanisms underlying inflammation offers opportunities for therapeutic intervention. During inflammation, matrix metalloproteinase (MMP) activity contributes to tissue remodeling and inflammatory signaling, and is regulated by tissue inhibitors of metalloproteinases (TIMPS). TIMP-1 and -2 have known roles in pain, but only in the context of MMP inhibition. However, TIMP-1 also has receptor-mediated cell signaling functions that are not well understood. Here, we examined how TIMP-1dependent cell signaling impacts inflammatory hypersensitivity and ongoing pain. We found that hindpaw injection of complete Freund's adjuvant (CFA) increased cutaneous TIMP-1 expression that peaked prior to development of mechanical hypersensitivity, suggesting that TIMP-1 inhibits the development of inflammatory hypersensitivity. To examine this possibility, we injected TIMP-1 knockout (T1KO) mice with CFA and found that T1KO mice exhibited rapid onset thermal and mechanical hypersensitivity at the site of inflammation that was absent or attenuated in WT controls. We also found that T1KO mice exhibited hypersensitivity in adjacent tissues innervated by different sets of afferents, as well as skin contralateral to the site of inflammation. Replacement of recombinant murine (rm)TIMP-1 alleviated hypersensitivity when administered at the site and time of inflammation. Administration of either the MMP inhibiting $\mathrm{N}$-terminal or the cell signaling C-terminal domains recapitulated the antinociceptive effect of full-length rmTIMP-1, suggesting that rmTIMP-1inhibits hypersensitivity through MMP inhibition and receptor-mediated cell signaling. We also found that hypersensitivity was not due to genotype-specific differences in MMP-9 activity or expression, nor to differences in 
cytokine expression. Administration of rmTIMP-1 prevented mechanical hypersensitivity and ongoing pain in WT mice, collectively suggesting a novel role for TIMP-1 in the attenuation of inflammatory pain.

Keywords: pain, thermal hyperalgesia, mechanical hypersensitivity, ongoing pain, matrix metalloproteinase, conditioned place preference

\section{INTRODUCTION}

Tissue inhibitors of matrix metalloproteinases (TIMPs) and matrix metalloproteinases (MMPs) are released during tissue damage to facilitate tissue remodeling through degradation and reorganization of the extracellular matrix (ECM) (Gardner and Ghorpade, 2003; Nagase et al., 2006; Ries, 2014). During this process MMPs also engage an inflammatory response through proteolytic maturation of cytokines, and both of these activities are regulated through a 1:1 stoichiometric interaction with one of four tissue inhibitors of metalloproteinases (TIMP-1, -2, -3, -4) (Huang et al., 2011). The interaction between MMPs and TIMPs is tightly controlled, but research has shown that during tissue damage, imbalance between MMPs and TIMPs can lead to pathological conditions such as arthritis, multiple sclerosis, Parkinson's Disease, cancer, and even chronic pain (Nakagawa et al., 1994; Nagase et al., 1999; Kouwenhoven et al., 2001; Yang et al., 2002; Brew and Nagase, 2010). Studies examining the role of MMPs in pain specifically have shown that increased MMP-2 and -9 activity contribute to increased pain-related behavior in response to injury that can be reversed by MMP antagonism (Kawasaki et al., 2008; Ji et al., 2009; Li et al., 2016; Remacle et al., 2018). These findings contributed, in part, to the development of several small molecule drugs that directly target and inhibit MMP activity. However, more than 50 clinical trials examining the efficacy of these drugs were discontinued due to the emergence of adverse events, including musculoskeletal pain (Cathcart and Cao, 2015; Martinho et al., 2016). While the results of these trials indicated that specific targeting of MMP activity alone is not an effective strategy for pain treatment, they also suggest that additional mechanisms related to MMP activity may contribute to pain and its inhibition, and that endogenous inhibitors of MMPs, such as TIMP-1, may attenuate pain-related behavior.

TIMP-1 is best characterized as an inhibitor of MMP activity. Indeed, TIMP-1 regulates 14 of the 24 known MMPs (Baker et al., 2002; Gardner and Ghorpade, 2003; Nagase et al., 2006; Kawasaki et al., 2008), and has been shown to prevent the development of mechanical and thermal hypersensitivity following nerve damage (Kawasaki et al., 2008; Martinho et al., 2016). However, this identified role was characterized purely in the context of MMP inhibition. TIMP-1 inhibits MMP activity through the binding of its N-terminus with the targeted MMP, resulting in chelation of $\mathrm{Zn}^{2+}$ from the enzyme active site (Gomis-Rüth et al., 1997). Interestingly, there is now mounting evidence that the C-terminal domain can bind to membrane bound receptors, including CD63 (Nagase et al., 2006). The binding of TIMP-1 to CD63 engages intracellular signaling events that allow TIMP-1 to function as a trophic factor and initiate cellular migration and differentiation (Gardner and Ghorpade, 2003; Jourquin et al., 2005; Thorne et al., 2009; Moore and Crocker, 2012; Claycomb et al., 2013). Because TIMP-1 and MMPs can be up-regulated simultaneously during tissue damage and repair, such as in peripheral nerve injury (Parkitna et al., 2006; Huang et al., 2011; Kim et al., 2012; Yokose et al., 2012), disentangling how TIMP-1 regulates tissue remodeling/repair from the induction of pain, per se, is challenging.

Inflammation is a core component of the nerve injury process (Tracey and Walker, 1995), and, in general, is a significant predictor of pain chronicity (Tal, 1999; Kehlet et al., 2006; Chapman and Vierck, 2017). Therefore, we used a model of cutaneous inflammation to examine the effects of TIMP-1 signaling on pain in the absence of frank tissue damage. We found that hindpaw injection of complete Freund's adjuvant (CFA) induced TIMP-1 expression in keratinocytes prior to the emergence of hypersensitivity in wildtype (WT) mice. Behavioral assessment of the role of TIMP-1 in inflammatory hypersensitivity demonstrated that TIMP-1 knockout (T1KO) mice exhibited robust hypersensitivity to stimulation of tissues local and distal to the site of inflammation that was prevented by administration of full length and truncated constructs of recombinant murine (rm)TIMP-1. These results also suggested that cell-signaling mechanisms may also contribute to the antinociceptive effects of TIMP-1. Finally, we found that the administration of rmTIMP-1 prevented ongoing inflammatory pain and evoked mechanical hypersensitivity in WT mice, collectively suggesting that TIMP-1 regulates the algogenic properties of inflammation and that TIMP-1 may be a target for improving pain management.

\section{MATERIALS AND METHODS}

\section{Animals}

Experiments were conducted using 8-12-week-old (2030 g) male WT (C57BL/6; Jackson Laboratories, Bar Harbor, ME) and T1KO mice that were group housed (4 mice/cage), and maintained in a temperature-controlled environment on a $12 \mathrm{~h}$ light-dark cycle with free access to food and water. TIMP-1 knockout (T1KO) mice (Lee et al., 2005) were backcrossed onto a C57BL/6 background for greater than 13 successive generations and bred in-house as a homozygous line (Crocker et al., 2006). All studies were approved by the UConn Health Institutional Animal Care and Use Committee and treated in accordance with published NIH standards. 


\section{Complete Freund's Adjuvant (CFA)}

To produce an acute, local inflammatory response, we subcutaneously (s.c.) injected the right hindpaw of mice with a diluted emulsion of CFA ( $1: 1$ in sterile $\mathrm{H}_{2} \mathrm{O} ; 10 \mu \mathrm{L}$ vol; Sigma, St. Louis, MO). To assess primary hypersensitivity (i.e., at the site of inflammation) we administered CFA into the glabrous skin or ventral surface of the right hindpaw. Conversely, secondary hypersensitivity was assessed in hairy skin that was adjacent or contralateral to the site of inflammation. All samples were compared to naïve controls because in a pilot experiment we found that vehicle injection alone caused increased sensitivity in T1KO mice (Supplementary Figure S1). While this result is interesting and suggests that subtle perturbations cause robust alterations in sensory thresholds, adding saline-treated mice confounds our ability to examine inflammatory hypersensitivity. Therefore, to interpret the effects of inflammation per se, naïve mice were used as comparison controls. The literature is also mixed on the use of vehicle controls in experiments using CFA, and our experiments are in line with previously published work (Allchorne et al., 2005; Jankowski et al., 2012; Imbe and Kimura, 2017).

\section{Recombinant Murine TIMP-1 Administration}

$\mathrm{WT}$ and T1KO mice received injections (s.c.) of recombinant murine (rm)TIMP-1 $(10 \mathrm{ng} / \mu \mathrm{L}, 10 \mu \mathrm{L}$; R\&D Systems; Minneapolis, MN) immediately following CFA injection $(10 \mu \mathrm{L})$ into the right hindpaw. In subsequent experiments, T1KO mice received equimolar concentrations of the truncated C-terminus peptide (TIMP-1(C); $6.3 \mathrm{kDa}$; Peptide 2.0 Inc., Chantilly, VA) that retains cell signaling function or the truncated $\mathrm{N}$-terminus peptide (TIMP-1(N); $20 \mathrm{kDa}$; Abcam, Cambridge, United Kingdom) that retains MMP-inhibitory function and no cell-signaling ability, immediately following CFA injection.

\section{von Frey Testing}

All mice were place into transparent Plexiglas chambers (radius $=32 \mathrm{~mm}$, height $=108 \mathrm{~mm}$ ) on an elevated mesh screen and were allowed to acclimate for a minimum of $1 \mathrm{~h}$ before testing. To assess mechanical sensitivity, the plantar surface of the right hindpaw was stimulated using von Frey filaments using the up-down method previously described (Dixon, 1980). Nocifensive responses were counted as robust flexion responses, paw shaking, or paw licking and subtracted from individual baseline threshold to account for inter-subject variability. Data are presented as paw withdrawal thresholds (PWT; in grams).

\section{Thermal Hyperalgesia}

Thermal hyperalgesia to radiant heat was assessed using a Hargreaves apparatus (Harvard Apparatus; Holliston, MA) (Hargreaves et al., 1988). Briefly, all mice were placed in transparent Plexiglas chambers (radius $=32 \mathrm{~mm}$, height $=108 \mathrm{~mm}$ ) on top of a framed glass panel and were allowed to acclimate for a minimum of $1 \mathrm{~h}$ before testing. Following the acclimation period, an infrared (IR) beam was aimed at the plantar surface of each hindpaw in an alternating fashion. The intensity of the IR beam was chosen to produce average baseline paw withdrawal latency (PWL) of 15-20 s. Stimuli were presented 5 times in an alternating fashion between each hindpaw with 5 min intervals between successive stimulus exposures. A $30 \mathrm{~s}$ exposure cutoff was employed to prevent tissue damage. PWLs collected from each paw were then averaged and analyzed.

\section{Conditioned Place Preference}

Conditioned Place Preference (CPP) was used to assess ongoing pain in WT mice. A 3-day single trial protocol was used. On day 1 , all mice freely explored a 3-chamber CPP box for $15 \mathrm{~min}$ prior to injection with CFA or saline. Preconditioning (baseline) behavior was analyzed using automated software (ANYMAZE, Stoelting) to ensure there were no baseline differences in the time spent in any of the chambers. On day 2 (conditioning day), all mice received an intrathecal (i.t.) injection of saline $(5 \mu \mathrm{L}$ volume) under isoflurane anesthesia and upon waking (within $2 \mathrm{~min}$ ) were confined into the pre-assigned pairing chamber for $30 \mathrm{~min}$. They were then returned to their home-cages for $4 \mathrm{hr}$. All mice then received an intrathecal (i.t.) injection of clonidine ( $2 \mu \mathrm{g} / \mu \mathrm{L} ; 5 \mu \mathrm{L}$ volume) through lumbar puncture and upon waking were confined to the opposite pairing chamber for $30 \mathrm{~min}$. Vehicle and clonidine paired chambers were randomly assigned and counterbalanced between animals. On day 3 (test day), mice were returned to the CPP apparatus and allowed to freely explore all chambers across $15 \mathrm{~min}$. The total time spent in each chamber was assessed using automated software (ANYMAZE). Conditioning day was 20-24 h following CFA injection as previous research indicates ongoing pain is observed at this time-point (Okun et al., 2011; He et al., 2012). A total of 17 mice were used, 8 were treated with rTIMP-1 and 9 with vehicle.

\section{Tissue Collection}

Mice were anesthetized with a lethal dose of ketamine and xylazine mixture $(90 / 10 \mathrm{mg} / \mathrm{kg}$, respectively) and intracardially perfused with ice cold $0.9 \%$ saline prior to the dissection and collection of tissues. Tissues were collected following the completion of behavior or at designated time-points for molecular analysis.

\section{Enzyme-Linked Immunosorbent Assay}

Protein was extracted from L2-L3 spinal cord, DRG and hairy skin and homogenized in ice-cold RIPA buffer/protease inhibitor cocktail and spun for $20 \mathrm{~min}$ at $4^{\circ} \mathrm{C}$ at $18,000 \mathrm{rcf}$. L2-L3 DRG were collected for analysis because axons from these DRG comprise the saphenous nerve, which innervates the hairy skin of the hindpaw, the site of CFA injection (McIlwrath et al., 2007; Lawson et al., 2008; Jankowski et al., 2009; Zimmermann et al., 2009; Vrontou et al., 2013; Laedermann et al., 2014). Each sample's total protein concentration was determined using Pierce BCA Protein Assay Kit (Thermo Fisher Scientific, Waltham, MA). The following Enzyme-Linked Immunosorbent Assay (ELISAs), TIMP-1 (R\&D Systems, Minneapolis, MN), MMP-9 (R\&D Systems; Minneapolis, MN), IL-6 (Invitrogen Carlsbad, CA), TNF- $\alpha$ (Thermo Fisher Scientific, Waltham, 
MA), IL-10 (Thermo Fisher Scientific, Waltham, MA), and IL$1 \beta$ (R\&D Systems, Minneapolis, MN) were run according to manufacturer's instructions. All samples were run in duplicate and absorbance ratios were read at $450 \mathrm{~nm}$.

\section{Immunohistochemistry}

Hairy skin, glabrous skin, and DRG from WT mice were excised and incubated in $0.06 \%$ brefeldin A (BFA) in serum free Hank's balanced salt solution (HBSS) for $20 \mathrm{~min}$ at room temperature. Half of the samples were incubated in inflammatory soup (IS) (10uM; bradykinin triacetate, histamine dihydrochloride, serotonin hydrochloride, prostaglandin E2 dissolved in normal cerebral spinal fluid, $\mathrm{pH}$ 6.0) or serum-free media (Kessler et al., 1992; Hachisuka et al., 2016). Incubation in IS or serum-free media occurred for $24 \mathrm{~h}$ (Kessler et al., 1992). Spinal cords from inflamed or naïve WT mice were isolated $24 \mathrm{~h}$ following CFA treatment or from designated naïve controls. Prior to tissue collection, mice were intracardially perfused with $0.06 \%$ BFA in $0.9 \%$ saline for $20 \mathrm{~min}$ and then perfused with $4 \%$ paraformaldehyde. All samples were post-fixed overnight in $4 \%$ paraformaldehyde (PFA), cryoprotected overnight in 30\% sucrose, and later embedded in Optimal Cutting Temperature (OCT). Samples were cut into $30 \mu \mathrm{m}$ cross sections using a cryostat. Tissue sections were briefly washed with sterile phosphate buffered saline (PBS) and incubated with staining buffer $(0.05 \%$ triton and $30 \%$ fetal bovine serum in PBS) solution for $40 \mathrm{~min}$ at room temperature. Slices were then incubated with primary unconjugated antibodies for $48 \mathrm{~h}$ at $4^{\circ} \mathrm{C}$. The following primary antibodies were diluted in staining buffer: monoclonal anti-mouse cytokeratin 14 (K14; Abcam, Cambridge, United Kingdom; 1:300 dilution), polyclonal anti-goat TIMP-1 (R\&D Systems; Minneapolis, $\mathrm{MN} ; 1: 300$ dilution), monoclonal anti-mouse microtubuleassociated protein 2 (MAP2; Millipore Sigma, Burlington, MA; 1:1000 dilution), and monoclonal anti-mouse primary conjugated-cy3 glial fibrillary acidic protein (GFAP; Abcam, Cambridge, United Kingdom; 1:500 dilution). Tissue slices were then incubated with secondary antibodies for $2-3 \mathrm{~h}$ at $4^{\circ} \mathrm{C}$. The following secondary antibodies were diluted in staining buffer: polyclonal rabbit anti-mouse Alexa-488 (Life Technologies, Carlsbad, CA, 1:000), polyclonal donkey antigoat Alexa-568 (Life Technologies, Carlsbad, CA,1:000), and polyclonal goat anti-mouse Alexa-568 (Life Technologies, Carlsbad, CA; 1:1000 dilution). Slides with DRG and spinal cord slices were incubated with $300 \mu \mathrm{m}$ DAPI prior to cover-slipping to visualize nuclei of satellite glial cells and astrocytes, respectively.

\section{MMP-9 Colorimetric Activity}

Protein was extracted from the hindpaw of WT and T1KO mice 1 day post-CFA injections (s.c., $10 \mu \mathrm{L}$ ) or from designated naïve controls for a high throughput screening of MMP-9 activity. Gelatinase activity was measured using the SensolyteGeneric MMP colorimetric assay kit (Anaspec, Fremont, CA) according to manufacturer's instructions. Samples were run in duplicate and end-point enzymatic activity was analyzed using a glutathione reference standard.

\section{RT-qPCR}

Total RNA was extracted from hairy skin collected from naïve and inflamed WT and T1KO mice 1 day following CFA injection using a RNeasy Mini Kit (Qiagen, Venlo, Netherlands). To quantify cutaneous TIMP-2 and TIMP-4 mRNA expression, equal amounts of cDNA were synthesized using the iScript cDNA Synthesis Kit (Bio-Rad Laboratories, Hercules, CA) and mixed with SsoAdvanced Universal SYBR Green Supermix (Bio-Rad Laboratories, Hercules, CA) and $2 \mu \mathrm{M}$ of both forward and reverse primers (see Table 1). GAPDH was amplified as an internal control. The threshold crossing value was noted for each transcript and normalized to the internal control. The relative quantitation of each transcript was performed using the $\Delta \Delta \mathrm{Ct}$ method (Livak and Schmittgen, 2001) and presented as fold change relative to naïve WT expression.

\section{Statistical Analysis}

All data were analyzed using one-way or mixed designs Analysis of Variance (ANOVA). Post hoc analyses were performed using Tukey's HSD, and statistical significance was determined using a $p<0.05$. Statistical analysis was performed using SPSS (Version $25)$. Since ANOVAs rely on linear relationships among data, and not all effects can be resolved using linear based statistical tests, we used trend analyses (e.g., contrasts) to test for significant nonlinear relationships in some of our behavioral analyses. An added benefit of this approach is that trend analyses are more robust than ANOVAs (Tabachnick and Fidell, 2007). Clonidine induced CPP was assessed using a 2-way repeated measures ANOVA with post hoc analysis of pre- vs. post- conditioning time spent in the clonidine paired chamber for each treatment group using Sidak's multiple comparisons test. Between groups analysis was performed on difference scores calculated as post-conditioning $(-)$ pre-conditioning time spent in the clonidine paired chamber.

\section{RESULTS}

\section{Cutaneous TIMP-1 Expression Is Upregulated Prior to the Onset of Inflammatory Hypersensitivity}

To determine whether cutaneous inflammation alters the expression of TIMP-1 in tissues along the peripheral sensory circuit, we injected diluted, emulsified CFA (10 $\mu \mathrm{L}$, s.c.) into the

TABLE 1 | Primer sequences for QPCR.

\begin{tabular}{|c|c|c|}
\hline Gene & Forward & Reverse \\
\hline Timp2 & $\begin{array}{l}5^{\prime} \text {-CCAGAAGAAGAGCCTG } \\
\text { AACCA-3' }\end{array}$ & $\begin{array}{l}\text { 5'-GTCCATCCAGAGGCAC } \\
\text { TCATC-3' }\end{array}$ \\
\hline Timp4 & $\begin{array}{l}\text { 5'-TGCAGAGGGAGAGC } \\
\text { CTGAA-3' }\end{array}$ & $\begin{array}{l}\text { 5'-GGTACATGGCACTGC } \\
\text { ATAGCA-3' }\end{array}$ \\
\hline Gapdh & $\begin{array}{l}\text { 5'-ATGAATACGGCTACAGCA } \\
\text { ACAGG-3' }\end{array}$ & $\begin{array}{l}\text { 5'-CTCTTGCTCAGTGTCCT } \\
\text { TGCTG-3' }\end{array}$ \\
\hline
\end{tabular}



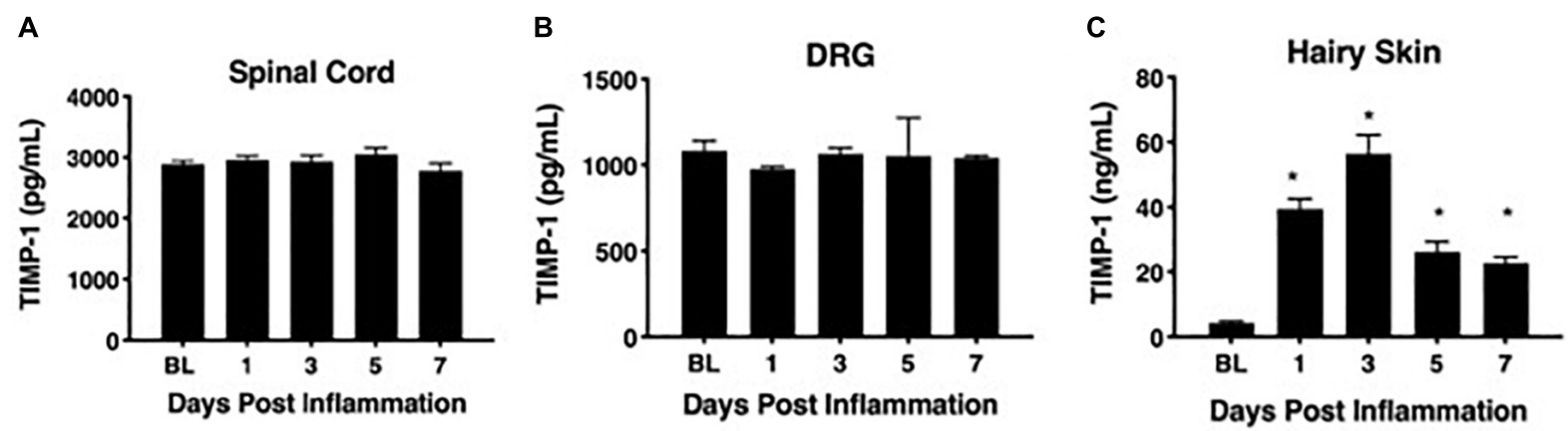

FIGURE 1 | Assessing TIMP-1 expression along peripheral nociceptive circuit following cutaneous inflammation. (A) Cutaneous inflammation does not alter overall TIMP-1 protein expression in lumbar spinal cord or (B) DRG, but does increase protein expression in panel (C) hairy skin. $n=4 /$ condition, ${ }^{*}$ indicate significant differences compared to naïve controls, $p<0.05$, and error bars depict SEM.

hairy skin of the ipsilateral hindpaw and collected spinal cord (SC; L2-L3), dorsal root ganglia (DRG; L2-L3), and hairy skin over the course of 7 days. As described in section "Materials and Methods," L2-L3 DRG were collected for analysis because the axons projecting from these DRG form the saphenous nerve, which innervates hindpaw hairy skin (McIlwrath et al., 2007; Lawson et al., 2008; Jankowski et al., 2009; Zimmermann et al., 2009; Vrontou et al., 2013; Laedermann et al., 2014). We found that inflammation did not alter the overall expression of TIMP-1 protein in SC or DRG, all Fs $>1.13, p>0.05$ (Figures 1A,B). However, we observed a significant increase in cutaneous TIMP-1 protein 1, 3, 5, and 7 days following CFA administration, $F(4,19)=37.54, p<0.01$ (Figure 1C). To confirm the above results, and to localize the cellular source of TIMP-1 expression, immunohistochemistry (IHC) was performed on DRG and skin samples incubated in vitro with or without inflammatory soup (IS) (Kessler et al., 1992), as well as spinal cords following in vivo inflammation. Because TIMP-1 is a releasable protein and, consequently, difficult to image, we utilized in vitro incubation of DRG and skin with IS to enhance our ability to capture TIMP-1 colocalization with markers of other cell types. Although overall TIMP-1 expression levels were unaltered in the spinal cord and DRG following inflammation, we found that TIMP-1 co-localized with glial fibrillary acidic protein (GFAP) expressing cells following inflammatory stimulation, suggesting that astrocytes (Figure 2A) and satellite glial cells (Figure 2B) appear to express TIMP-1 during inflammation (Huang et al., 2011; Welser-Alves et al., 2011). We also found that TIMP-1 expression was upregulated in K14-positive keratinocytes in both hairy and glabrous skin following inflammatory stimulation (Figure 2C; glabrous skin data not shown).

To associate the expression of cutaneous TIMP-1 with the development of mechanical hypersensitivity, we assessed PWT on the plantar surface of the hindpaw for 7 days following CFA injection into the dorsal, hairy skin. We found that TIMP-1 protein levels peaked 3 days following CFA administration (see Figure 1C), at a time when mice developed mechanical hypersensitivity, $F(2,12)=43.94, p<0.05$, (Figure 3). Together, these data indicate that cutaneous inflammation induces the expression of TIMP-1 in keratinocytes at the time of inflammation and prior to the onset of mechanical allodynia.

\section{Mice Lacking TIMP-1 Exhibit Hyperalgesia in Inflamed and Uninflamed Cutaneous Tissues}

To determine whether endogenous TIMP-1 expression is important for the normal progression of hypersensitivity, we used a global TIMP-1 knockout (T1KO) mouse strain. We first assessed behavioral responsiveness to radiant heat on the plantar surface of the hindpaw following s.c. administration of a diluted, emulsified CFA solution. We chose to use a diluted CFA solution because our preliminary experiments suggested that exposure to slight challenges significantly altered sensitivity in T1KO. To ensure that any potential differences in responding to inflammatory stimulation were not due to preexisting differences in sensory thresholds between mouse strains, we measured baseline responding to radiant heat and found no significant differences in PWL, $F(1,31)=0.47$, $p>0.05$ (Figure 4A). Interestingly, while we did not observe any significant differences in PWL between naïve and WT mice that received diluted (e.g., subthreshold) CFA, we did find that inflamed T1KO mice exhibited thermal hyperalgesia that persisted for 29 days in response to diluted CFA injection, all Fs $<2.30, p<0.05$ (Figure 4B).

Next, we assessed mechanical response thresholds (von Frey) following diluted CFA administration. Analysis of baseline responses to mechanical stimulation did not reveal any significant differences between genotypes $F(1,36)=0.34$, $p<0.05$ (Figure 4C). We did find that CFA administration reduced mechanical response thresholds in both genotypes $F$ ( 1 , $34)=17.61, p<0.01$. However, T1KO mice exhibited greater mechanical hypersensitivity 1 day following CFA treatment, compared to WT controls, all Fs $<4.59, p<0.05$ (Figure 4D). Therefore, we concluded that the up-regulation of TIMP-1 following inflammation delays the onset of hypersensitivity, and that in the absence of TIMP-1, the normal development of hypersensitivity is altered. 


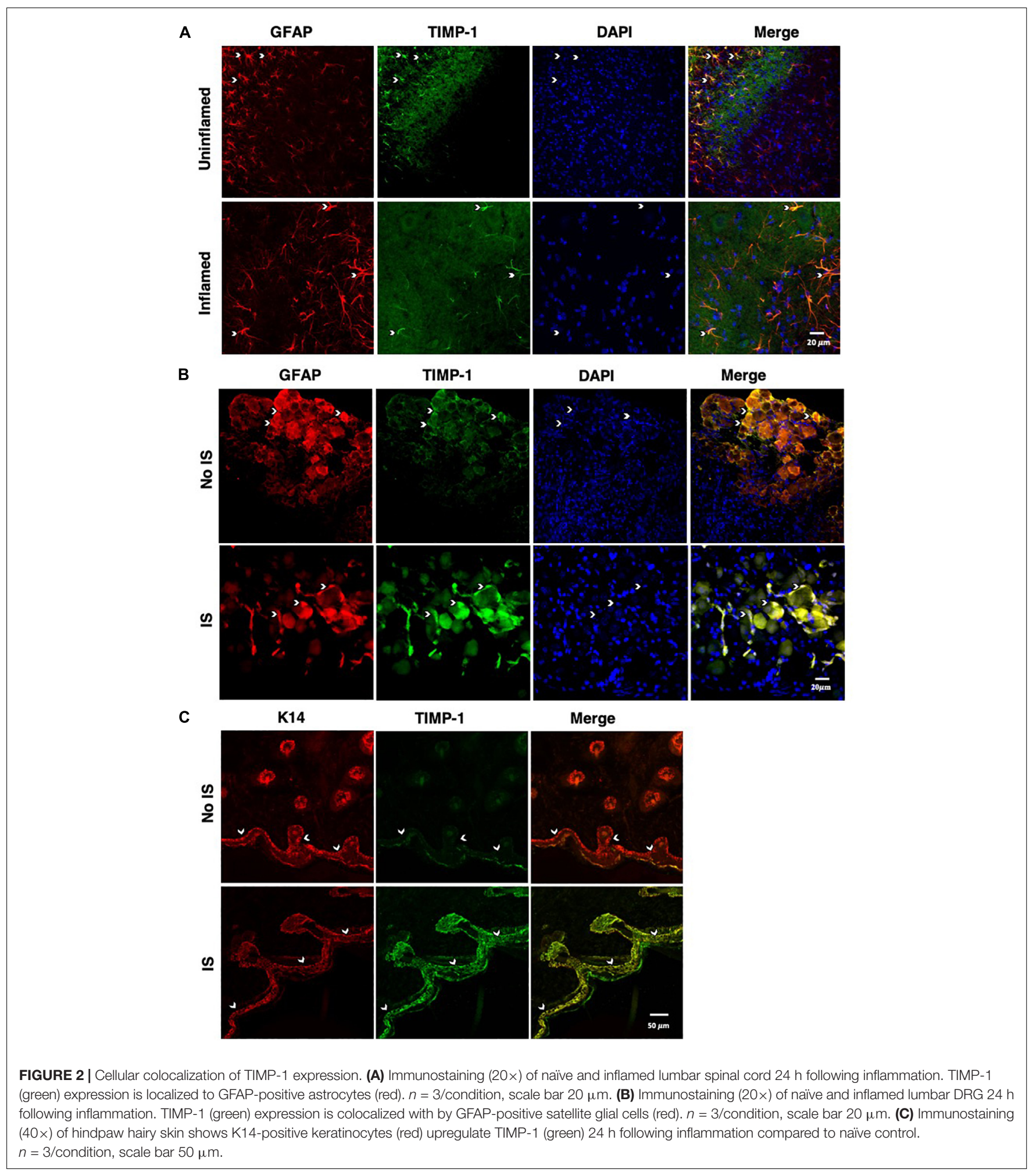

To determine whether the rapid emergence of inflammatory hypersensitivity in T1KO mice was due to compensatory expression of Timp 2 or Timp 4 mRNA, we examined cutaneous expression of each transcript in naïve and inflamed T1KO mice. We focused on Timp2 and Timp4 expression because,
TIMP-2 and -4 are soluble extracellular protease inhibitors that also share functional similarities with TIMP-1. TIMP3 , by contrast is quite different from the other TIMPs and is insoluble, tethered to the extracellular matrix, and is a more effective inhibitor of membrane-bound MMPs that 


\section{Mechanical Allodynia}

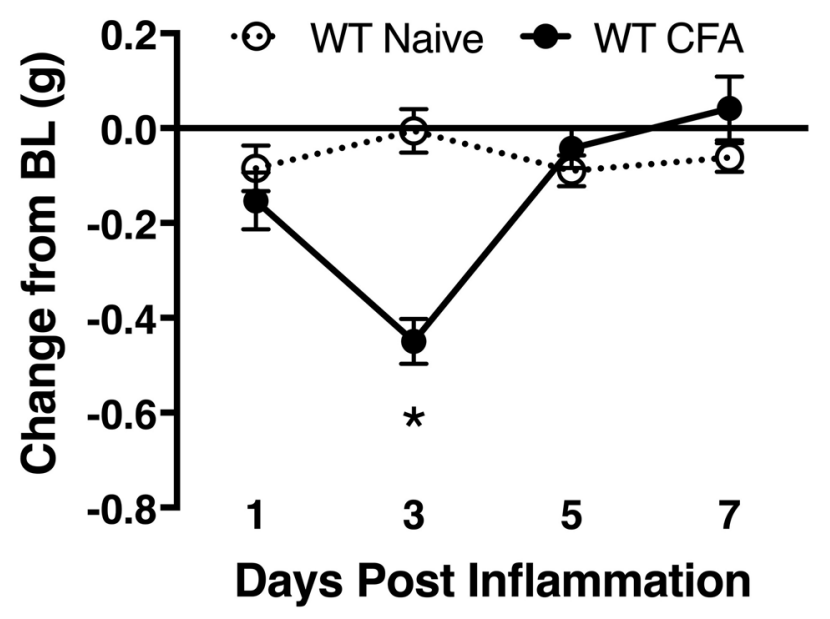

FIGURE 3 | Development of inflammatory hypersensitivity in WT mice. Assessment of mechanical hypersensitivity over 7 days following s.c. administration of CFA. Inflamed mice show a significant reduction in mechanical thresholds relative to naïve mice 3 days following inflammation ( $n=6 /$ condition). * indicate significant differences compared to naïve controls, $p<0.05$, and error bars depict SEM.

extracellular MMPs. Moreover, prior studies have documented some role for TIMP-2 in pain (Ji et al., 2009), and TIMP4 is known to inhibit MMP-2 and -9 (Ahmed et al., 2011; Grünwald et al., 2018), which also have identified roles in pain (Ji et al., 2009). Conversely, little is known about how TIMP-3 directly contributes to pain development or maintenance. Analysis of Timp 2 and Timp4 expression revealed no significant differences in the basal expression of either transcript in WT or T1KO mice. However, Timp2 and Timp4 expression decreased in $\mathrm{T} 1 \mathrm{KO}$ mice following inflammation (Figures 5A,B), suggesting that genetic deletion of TIMP-1 did not result in a compensatory response from other TIMPs, all $F s<11.65, p<0.01$.

Our current data demonstrate that cutaneous TIMP-1 is an early emergent protein following inflammation, and that the absence of TIMP-1 alters the normal development of hypersensitivity. Therefore, TIMP-1 signaling may have important implications for regulating the development of inflammatory hypersensitivity in tissue adjacent to the site of inflammation that is innervated by afferent terminals that are different from those that innervate inflamed skin. To test this possibility, we assessed the development of hypersensitivity in the glabrous skin following injection of diluted CFA into hairy skin in both $\mathrm{T} 1 \mathrm{KO}$ and WT following baseline assessment of sensitivity. Again, we observed no genotype-specific differences in baseline reactivity, and because of this consistent finding, we will no longer present data depicting baseline behavioral reactivity. Analysis using an ANOVA revealed that $\mathrm{T} 1 \mathrm{KO}$ mice, relative to WT mice, exhibited increased sensitivity to mechanical stimulation on the plantar surface of the hindpaw following inflammation of hairy skin that was not temporally dependent, all Fs $<4.41, p<0.05$, (Figures 6A,B). However, trend analyses revealed that inflamed T1KO mice exhibited increased inflammatory hypersensitivity 1 day following CFA treatment when compared to all other mice, $F(1,57)=11.55$, $p<0.01$ (Figure 6A).

To examine whether administration of recombinant murine (rm)TIMP-1 prevented inflammatory hypersensitivity, and the potential mechanism by which this effect occurs, separate groups of $\mathrm{T} 1 \mathrm{KO}$ mice received a single injection $(10 \mu \mathrm{L}$ vol) of recombinant full-length rmTIMP-1 [TIMP-1(FL)], the truncated $\mathrm{N}$ terminus peptide [TIMP-1(N)] that retains MMP inhibitory function but no cell signaling capacity, or the truncated C terminus peptide [TIMP-1(C)] that lacks MMP inhibitory capacity but retains its cell signaling function at the time of CFA administration. To limit the complexity of our experimental design, and to determine the optimal dose for the administration of each TIMP-1 construct, we conducted a pilot experiment using a small cohort of T1KO mice given 1,10 , or $100 \mathrm{ng} / \mu \mathrm{L}$ of TIMP-1 at the time of inflammation. We found that $10 \mathrm{ng} / \mu \mathrm{L}$ was effective at reducing inflammatory hypersensitivity (Supplementary Figure S2). We then administered a separate cohort of $\mathrm{T} 1 \mathrm{KO}$ mice $10 \mathrm{ng} / \mu \mathrm{L}$ of TIMP-1(FL), TIMP-1(N), or TIMP-1(C) at the time of CFA administration. Mechanical hypersensitivity was assessed $24 \mathrm{~h}$ later. While inflamed T1KO mice exhibited a significant reduction in mechanical thresholds, T1KO mice treated with the rmTIMP-1 peptide constructs did not. Moreover, we observed no significant differences in the response thresholds between mice given TIMP-1(FL), TIMP-1(N), or TIMP-1(C), all Fs $>4.54, p<0.01$ (Figure 6C), demonstrating that TIMP-1 attenuates inflammatory hypersensitivity through MMPdependent and MMP-independent signaling mechanisms.

The above data show that inflammation in one somatic region could lead to mechanical hypersensitivity in tissue distal to the site of inflammation, reminiscent of "mirror image pain" (Treede et al., 1992, 2015). To test this possibility, we inflamed one hindpaw and measured mechanical sensitivity on the opposite hindpaw for 7 days following CFA administration. We also examined whether treatment with rmTIMP-1 at the site and time of inflammation affected sensitivity. We found that inflamed $\mathrm{T} 1 \mathrm{KO}$ mice exhibited contralateral mechanical hypersensitivity over the course of 7 days following CFA-injection relative to WT mice (Figure 6D). Interestingly, this contralateral hypersensitivity was prevented by treatment with rmTIMP-1 in T1KO mice, $F(4,43)=5.52, p<0.05$ (Figure 6D).

\section{The Lack of TIMP-1 Does Not Alter the Expression of Local Inflammatory Molecules}

TIMP-1 is primarily known as a broad-spectrum MMP inhibitor, and because MMPs are known to contribute to hypersensitivity, we hypothesized that the absence of TIMP1 may cause hypersensitivity due to elevated activity and expression of cutaneous MMP-9 (Kawasaki et al., 2008; Brew and Nagase, 2010). Examination of hairy skin collected 1 day following CFA from WT and $\mathrm{T} 1 \mathrm{KO}$ mice demonstrated that 
A

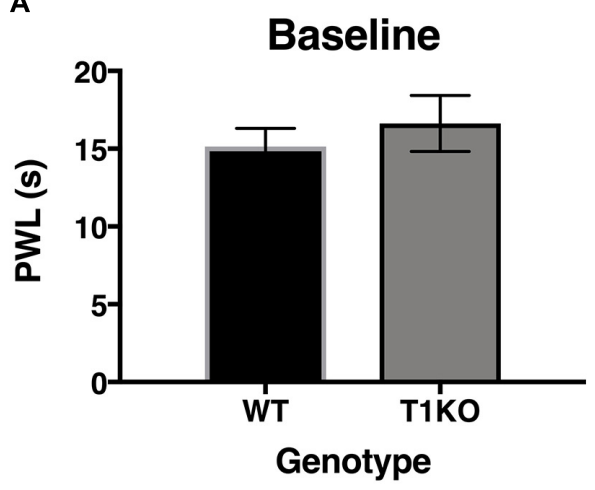

C

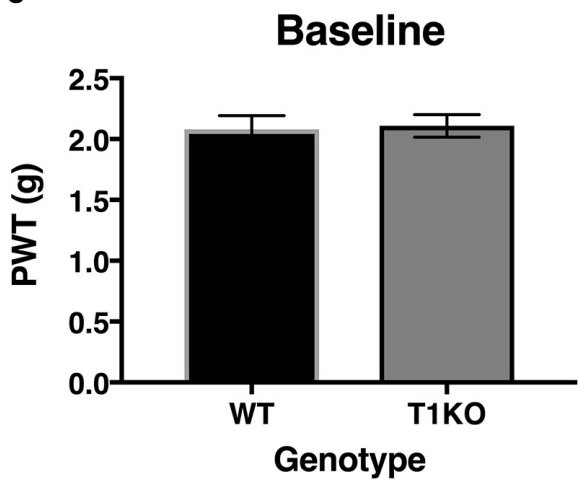

B Thermal Hyperalgesia

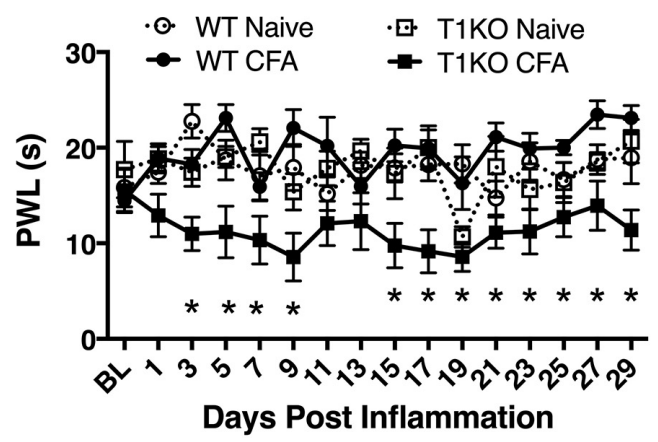

D

Mechanical Sensitivity

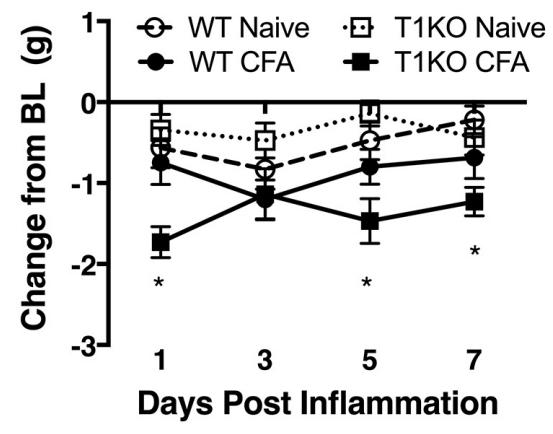

FIGURE 4 | Mice lacking TIMP-1 develop thermal and mechanical hypersensitivity following cutaneous inflammation. (A) No differences in baseline thermal PWL are exhibited between T1KO and WT mice ( $n=16 /$ condition). (B) Inflamed T1KO mice exhibit significantly reduced PWLs compared to inflamed WT mice and naïve WT and T1KO mice ( $n=8$ /condition). (C) Baseline assessment of mechanical PWTs revealed no genotypic differences between T1KO ( $n=20)$ and WT mice $(n=18)$. (D) T1KO mice develop significantly reduced PWTs 1 , 5, and 7 days following CFA administration compared to WT controls $(n=8-10 /$ condition). * $=$ signficantly different from mice in all other conditions, $p<0.05$, and error bars depict SEM.
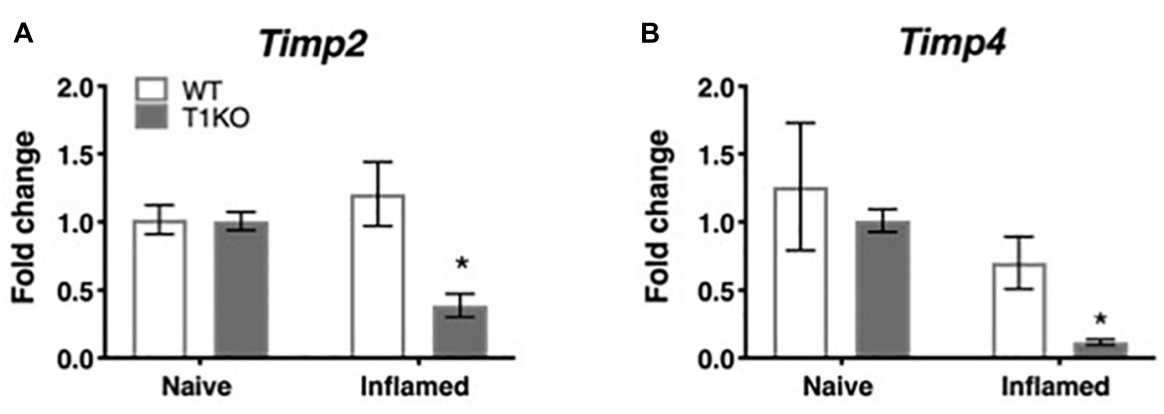

FIGURE 5 | Assessment of cutaneous Timp2 and Timp4 mRNA expression following inflammation. (A) Timp2 mRNA expression is decreased in T1KO mouse skin 1 day following inflammation relative to WT controls. (B) Timp4 mRNA expression is decreased 1 day following CFA compared to WT inflamed mice. $n=4 /$ condition, * indicate significant differences compared to naïve controls, $p<0.05$, and error bars depict SEM.

there was an inflammation-induced increase in both MMP9 expression and activity, all $F s>7.61, p<0.05$ but that these effects were not genotype-specific, all $F s<2.05$, $p>0.05$ (Figures 7A,B). The TIMP/MMP axis also regulates the proteolytic maturation of inflammatory molecules which can cause hypersensitivity (Pagenstecher et al., 1998). We next assessed whether the absence of TIMP-1 during inflammation caused elevated cytokine expression in the skin. Using ELISAs, we assessed the expression of cutaneous IL- $1 \beta$, IL- 6 , TNF- $\alpha$, and IL-10 at 1 day following CFA-injection. Analysis revealed an inflammation-induced increase in IL- $1 \beta$ and IL- 6 expression, but this increase in expression was not different between genotypes, 


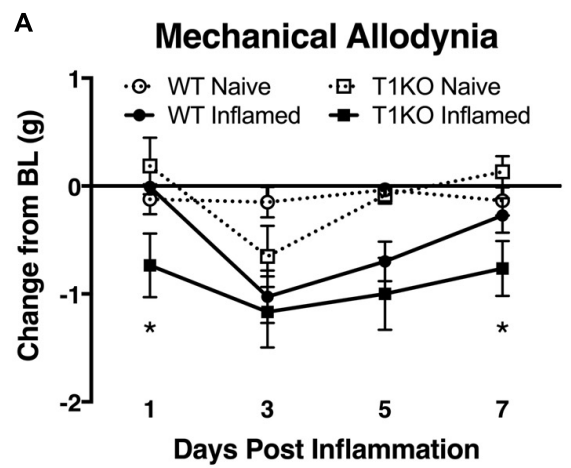

C

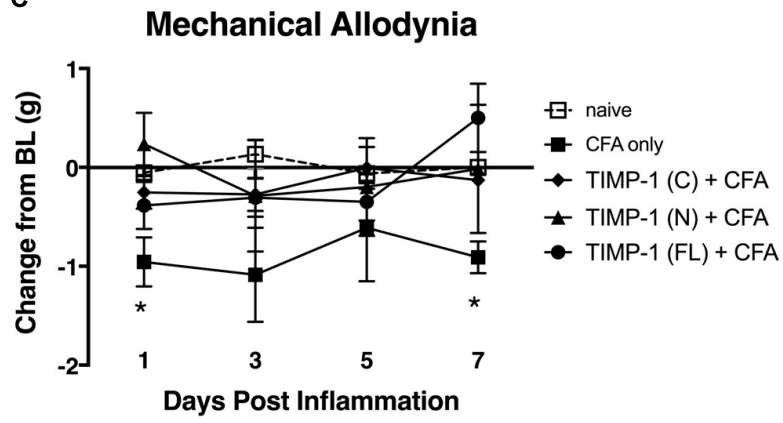

B

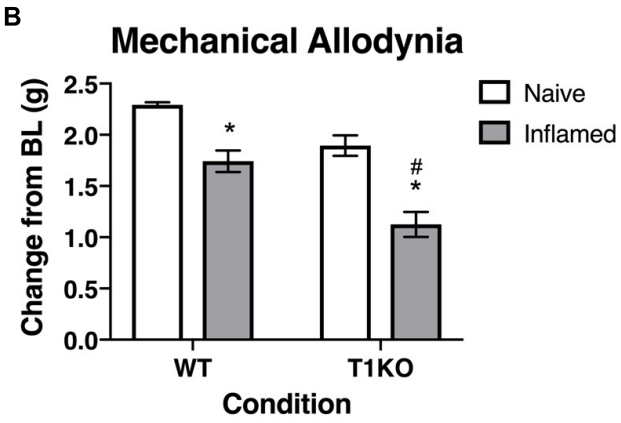

D

Dechanical allodynia

- WT Naive $\because$ T1KO Naive

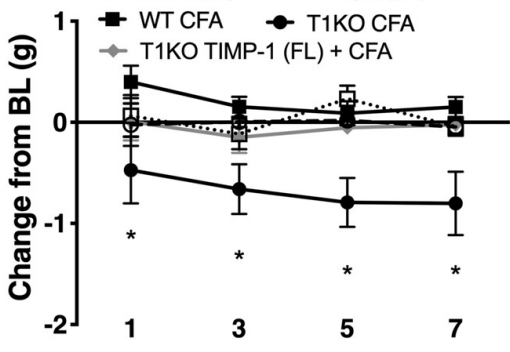

Days Post Inflammation

FIGURE 6 | Mice lacking TIMP-1 show increased sensitivity in non-inflamed tissues. (A) Injection of CFA into the hairy skin causes mechanical hypersensitivity on the plantar surface of the paw to develop 1 day following inflammation in T1KO, but not WT, mice. (B) Graph depicting mechanical responsiveness following inflammation collapsed across time. Inflamed T1KO mice greater mechanical sensitivity overall following cutaneous inflammation. (C) Administration of TIMP-1(FL), TIMP-1(N), or TIMP-1(C) into the hairy skin at the time of inflammation prevents the development of mechanical hypersensitivity in T1KO mice. (D) Hindpaw administration of CFA produces mechanical hypersensitivity on the paw contralateral to inflammation in T1KO relative txo WT mice. Treatment with rmTIMP-1 attenuated contralateral hypersensitivity in T1KO mice. PWT are presented as change from baseline. $n=8 /$ condition, * represent significant differences relative to naïve controls, $p<0.05$, and error bars depict SEM. " significantly different from WT mice.

all $F s>12.94, p<0.05$ (Figures 7C,D). In comparison, analysis of TNF- $\alpha$ and IL-10 did not reveal any significant differences following inflammation, all $F s<4.64, p>0.05$ (Figures 7E,F). These data suggest that TIMP-1 does not affect the emergence of hypersensitivity through differences in inflammatory cytokine expression.

\section{Administration of Recombinant TIMP-1 Attenuates Ongoing Pain in WT Mice}

Previous experiments demonstrate that the administration of rmTIMP-1 attenuates evoked mechanical and thermal hypersensitivity in $\mathrm{T} 1 \mathrm{KO}$ mice. Here, we examined whether the administration of rmTIMP-1 also attenuated ongoing pain in WT mice using CPP as previous described (King et al., 2009; Ellis and Bennett, 2013). Analysis of pre- compared to post-conditioning time spent in the conditioning chamber indicate an effect of drug, $F(1,30)=7.269, p<0.05)$, and post hoc analysis confirmed an increase in post-conditioning time spent in the clonidine paired chamber compared to pre-conditioning time in vehicle treated mice $(p<0.01)$ but not in rmTIMP-1 treated mice $(p>0.05)$ (Figure 8). These observations indicate that WT mice administered CFA and TIMP-1 did not demonstrate clonidine-induced CPP (Figure 8). Because clonidine only produces CPP in the state of injury (King et al., 2009), these results further suggest that treatment with rmTIMP-1 attenuated ongoing inflammatory pain.

\section{DISCUSSION}

The balance between TIMPs and MMPs is important for maintaining tissue homeostasis and preventing pathological conditions. Following tissue damage and inflammation, TIMP1 is expressed in a variety of cell types that can modulate neuronal function and wound healing, including, astrocytes, oligodendrocytes, Schwann cells, endothelial cells, mast cells, and keratinocytes (Fagerberg et al., 2010; Yokose et al., 2012; Claycomb et al., 2013). Because TIMP-1 is broadly expressed, TIMP-1 may also regulate neuroinflammation and neuropathic pain (Dhar et al., 2006; Kawasaki et al., 2008; Ji et al., 2009; Huang et al., 2011). Although the predominant view is that TIMP-1 exerts these functions by inhibiting MMPs, emerging evidence suggests that TIMP-1 may also facilitate these functions by binding cell-surface receptors and mediating their subsequent 


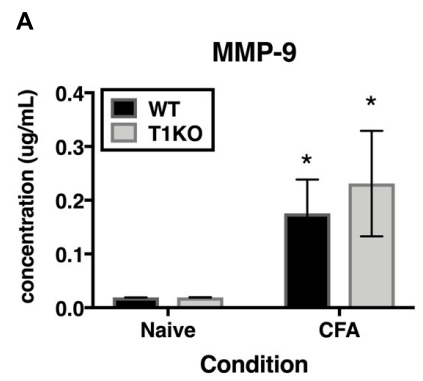

D

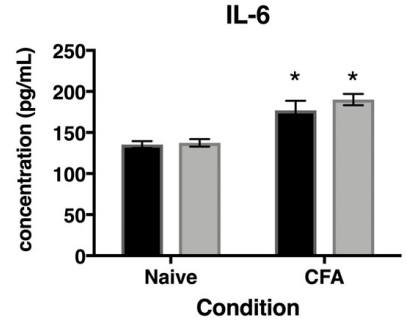

B

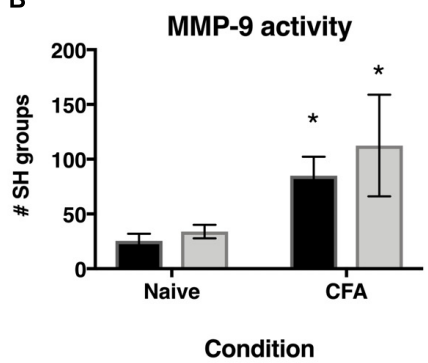

E

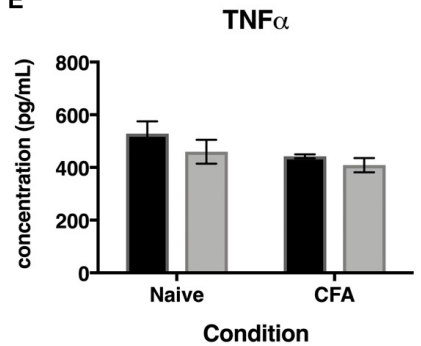

C

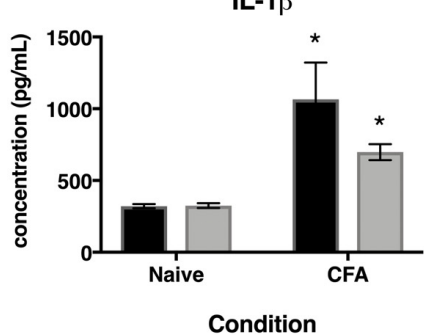

$\mathbf{F}$

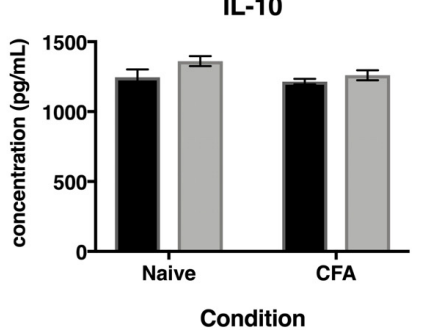

FIGURE 7 | Inflammation does not alter pro-inflammatory molecules in a genotype-specific manner. (A) Cutaneous inflammation significantly increases MMP-9 protein expression in WT and T1KO skin 1 day following CFA administration ( $n=7 /$ condition). (B) Cutaneous inflammation increases MMP-9 activity in WT and T1KO hairy skin 1 day following CFA administration. (C) Cutaneous inflammation significantly increases IL-1 $\beta$ protein expression in WT and T1KO hairy skin 1 day following inflammation. (D) Cutaneous inflammation significantly increases IL-6 protein expression in WT and T1KO hairy skin 1 day following CFA administration.

(E) Cutaneous inflammation does not affect expression of TNF- $\alpha$ following CFA administration. (F) Cutaneous inflammation does not affect expression of IL-10 protein in WT and T1KO skin following CFA administration. $n=4$ /condition, ${ }^{*}$ represent significant differences relative to naïve controls, $p<0.05$, and error bars depict SEM.

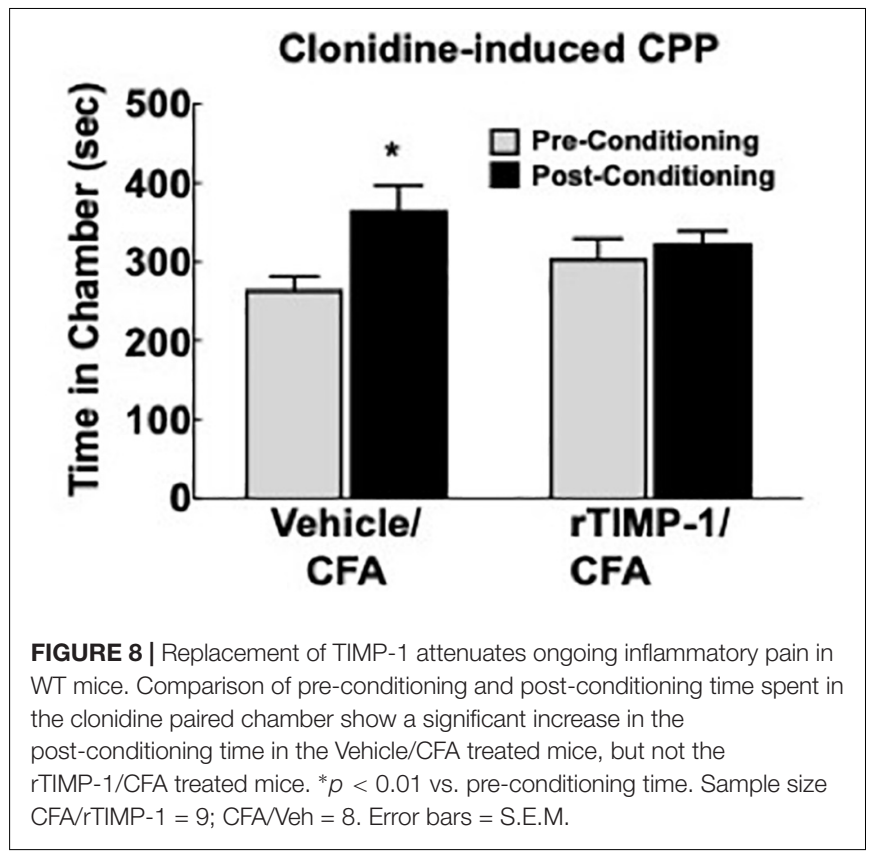

downstream signaling pathways (Dhar et al., 2006; Toricelli et al., 2013; Thevenard et al., 2014; Nicaise et al., 2019). Therefore, the present set of studies was designed to investigate the role of TIMP-1 in the development of inflammatory hypersensitivity and the mechanisms of its action.
To determine how inflammation affected the expression of TIMP-1 in tissues proximal and distal to the site of CFAinjection, we examined TIMP-1 expression in skin, DRG, and spinal cord over the course of 7 days. While we did not observe changes in TIMP- 1 expression in the DRG or spinal cord, we did localize its expression to GFAP-positive cells, suggesting that TIMP-1 is expressed by satellite glial cells and astrocytes, respectively. When we examined skin, we found that CFA induced an 8.27-fold increase in TIMP-1 protein expression within $24 \mathrm{~h}$ of inflammation, and that this temporal upregulation in TIMP-1 was observed in basal keratinocytes. Given that keratinocytes augment nociceptive signaling through the release of neuroactive molecules (Baumbauer et al., 2015; Moehring et al., 2018), the release of TIMP-1 from keratocytes may attenuate pronociceptive behavior caused by inflammation (Fagerberg et al., 2010; Yokose et al., 2012; Edqvist et al., 2015). To test this hypothesis, we examined the temporal expression pattern of TIMP-1 in relationship to the development of cutaneous hypersensitivity in WT mice. We found that the largest change in TIMP-1 expression, within $24 \mathrm{~h}$ of injection, preceded the onset of cutaneous hypersensitivity, and that at 3 days when TIMP-1 expression peaked, behavioral sensitivity was the greatest in WT mice. This result suggests that peak TIMP-1 expression may in some way signal the onset of hypersensitivity. Alternatively, it is also possible that the relationship between TIMP-1 expression and the onset of hypersensitivity is determined by a relative change in expression between two consecutive 
time points. In the case of the current experiments the largest change in expression is observed between baseline and $24 \mathrm{~h}$ following inflammation, where we observe an 8.27-fold change in expression. Conversely, we only detect a 1.43 fold increase in expression between Day 1 and Day 3, implying that it is not the absolute level of TIMP-1 expression, per se, that contributes to the delay in hypersensitivity, but rather the extent to which TIMP-1 expression changes relative to previous levels of expression over time. Consequently, it may be possible that as TIMP-1 expression increases during the first $24 \mathrm{~h}$ of inflammation the emergence of pain-related behavior is attenuated. However, the overall change in TIMP1 expression over the next $48 \mathrm{~h}$ is no longer sufficient to prevent the emergence of hypersensitivity. Supporting this, we found that the replacement of recombinant TIMP-1 within $24 \mathrm{~h}$ of CFA-injection, which causes a significant increase in expression from baseline expression in the skin, prevented the onset of hypersensitivity in mice lacking TIMP-1. Taken together, these data suggest that the immediate induction and release of TIMP-1 from basal keratinocytes attenuates inflammatory hypersensitivity.

If the release of TIMP-1 is important for delaying the onset of inflammatory hypersensitivity, it is also possible that hypersensitivity is exacerbated in the absence of TIMP1. To test this, we compared mechanical hypersensitivity and thermal hyperalgesia at the site of inflammation in WT and $\mathrm{T} 1 \mathrm{KO}$ mice. While we observed a robust reduction in mechanical thresholds in WT and T1KO mice, hypersensitivity persisted for a longer duration in $\mathrm{T} 1 \mathrm{KO}$ mice relative to WT controls. Interestingly, when we examined thermal hyperalgesia, we observed a significant reduction in PWT in T1KO mice, while WT mice appeared to be unaffected. This result suggests that TIMP-1 may differentially regulate the processing of thermal and mechanical stimulation. In particular, in WT mice, the presence of TIMP-1 appears to delay the onset and persistence of mechanical sensitivity while having no effect on thermal reactivity during mild inflammation. More broadly, others have argued that mechanical sensitivity is a hallmark sign of pathological pain states (Treede et al., 1992, 2015), and our results imply that the dysregulation in TIMP-1 signaling may contribute to this process by influencing hypersensitivity to mechanical stimulation. Importantly, our observed results were not due to a compensatory response in expression of Timp2 or Timp4, and both transcripts are expressed at similar levels in both WT and T1KO mice in the naïve state. We did observe that expression of both Timp2 and Timp4 decreased following inflammation further suggesting a role for TIMP-2 (Kawasaki et al., 2008; Ji et al., 2009), and possibly TIMP4 , in the emergence of hypersensitivity. Specifically, decreased expression and activity of TIMP-2 and -4 may encourage the emergence and persistence of hypersensitivity. How each TIMP functionally differs in this capacity is the subject of ongoing work.

Given that thermal hyperalgesia and prolonged mechanical hypersensitivity were observed in $\mathrm{T} 1 \mathrm{KO}$, but not WT mice, following mild inflammation, our data suggest that the absence of TIMP-1 increases susceptibility to subtle perturbations that would otherwise be considered innocuous. Indeed, we found that injections of normal physiological saline produced mechanical hypersensitivity in $\mathrm{T} 1 \mathrm{KO}$ mice, which was not observed in WT controls. This hypersensitivity could be due to a number of plausible factors, including hypertonicity in a TIMP-1 deficient system (Steinbrocker et al., 1953; Giesler and Liebeskind, 1976; Hylden and Wilcox, 1983; Sutaeg Hwang and Wilcox, 1986), the disruption of cutaneous integrity from needle insertion, cutaneous distention following injection, or the induction of some inflammatory process. Whatever the cause of hypersensitivity is following saline injection, it is tempting to postulate that when TIMP-1 is not present, the physiological processes in the periphery become dysfunctional and sensory stimulation is amplified.

Pathological pain is also characterized by increased sensitivity in tissues adjacent to, and distal from, the site of inflammation, as is the case with "mirror image" pain (Shir and Seltzer, 1991; Maleki et al., 2000). When we assessed sensitivity in tissue adjacent to the site of inflammation and that are innervated by different sets of afferent terminal endings (e.g., hairy vs. glabrous skin), we found that T1KO mice exhibited mechanical hypersensitivity. Interestingly, we observed hypersensitivity in the uninflamed (contralateral) hindpaw, and the sensitivity occurred in a different dermatome from the inflamed dermatome. Administration of rmTIMP-1 at the site of inflammation alleviated hypersensitivity on both the inflamed and uninflamed paws. These data suggest that inflammationinduced TIMP-1 expression occurs in a coordinated fashion that influences the normal progression of inflammatory sensitivity in both inflamed and uninflamed tissues, which may attenuate afferent input and prevent the development of central sensitization.

TIMP-1 is well known as an inhibitor of MMPs, and we know that MMPs contribute to pain following various injury and inflammatory conditions (Kawasaki et al., 2008; Remacle et al., 2018). We hypothesized that disrupting the balance between TIMP and MMP expression and activity would exacerbate hypersensitivity in T1KO mice due to elevated MMP activity and pro-inflammatory cytokine expression. However, we did not detect any genotype-specific differences in the activity of MMP-9 or pro-inflammatory cytokines proximal to the site of CFA administration. Prior work indeed shows that the inhibition of MMPs reduces pain (Kawasaki et al., 2008; Ji et al., 2009), and our current results demonstrate that administration of the N-terminal domain of TIMP-1, the domain responsible for MMP inhibition, attenuated hypersensitivity. We also show that administration of TIMP-1(C), the domain responsible for engaging receptor-mediated cell signaling events (Jung et al., 2006; Toricelli et al., 2013; Takawale et al., 2017; Nicaise et al., 2019), also attenuates hypersensitivity following inflammation, suggesting that TIMP-1 may also delay the emergence of hypersensitivity through a novel receptor-mediated mechanism. Consequently, TIMP-1 may attenuate the development of pain through both pathways. This latter point may help to explain, at least in part, why small molecule inhibitors of MMP activity have limited efficacy (Cathcart and Cao, 2015). By understanding 
how both subdomains alleviate hypersensitivity, we may be able to effectively manage pain progression.

While our results show that TIMP-1 attenuates pain and hypersensitivity through both MMP inhibition and receptormediated signaling, the precise mechanisms by which TIMP-1 acts are not known. Our data suggest that the amount of TIMP1 present at the site of inflammation may determine functional outcomes, which is consistent with previously published work (Grünwald et al., 2018). Moreover, inflammation affects a variety of cells types that are mobilized to encourage wound repair, including macrophages, dendritic cells, $\mathrm{B}$ cells, and $\mathrm{T}$ cells. Not only is TIMP-1 released by immune cells, but it is released by endothelial cells as well, and this release of TIMP-1 increases vascular permeability (Shubayev and Strongin, 2018), allowing cells to infiltrate injured tissue during the repair process. Interestingly, the ability of TIMP-1 to increase vascular permeability is thought to occur through MMP-independent signaling mechanisms (Stetler-Stevenson, 2008). In addition to TIMP-1, all of these cells are known to release the antiinflammatory cytokine interleukin-10 (IL-10) (Couper et al., 2008), and IL-10 increases the production of TIMP-1 (Koscsó et al., 2013). There are a large number of cells that can produce TIMP-1, including mast cells within the skin. Because mast cells have a well-characterized role in driving allergic sensitivity and the release of cytokines involved in itch and pain (Galli and Tsai, 2012; Mukai et al., 2018), the release of mast cellderived TIMP-1 producing ant nociception seems at odds with our current results. However, research has shown that mast cells contribute to the emergence of analgesia (Kalesnikoff and Galli, 2008; Cui et al., 2018), and this analgesic/antinociceptive response may result from the release of TIMP-1 in an IL-10dependent manner. It is important to note that we did not observe a genotype-specific changes in IL-10 concentration in the skin following inflammation, but this may have occurred because IL-10 is upstream of TIMP-1 and deletion of TIMP-1 may not impact IL-10 release.

Once released into the extracellular space, TIMP-1 can potentially attenuate pain through multiple pathways. One such pathway is through the inhibition of MMPs, which not only affects their ability to produce pain, but also prevents the release of mature nerve growth factor (NGF) and brain-derived neurotrophic factor (BDNF) (Shubayev and Strongin, 2018), both of which have known roles in pronociceptive signaling (Woolf et al., 1994; Andreev et al., 1995; Bennett et al., 1998; Zhao et al., 2006; Melemedjian et al., 2010, 2013). Consequently, the antinociceptive effects of TIMP-1 may occur through inhibition of NGF, BDNF, or other algogenic molecules.

Similar processes may not only occur within the skin, but also the DRG and spinal cord as well. Our results, along with the work of others (Thalakoti et al., 2007; Vause and Durham, 2010), show that TIMP-1 is expressed by SGCs in the DRG, suggesting that the release of TIMP-1 by SGCs may attenuate the effects of pronociceptive molecules, such as NGF and BDNF, and affect the way in which afferent cell bodies respond to inflammation. TIMP-1 is expressed in a variety of cell types in the spinal cord with known roles in pain processing, including astrocytes and microglia. In general, the release of
TIMP-1 in the spinal cord appears to abrogate the effects of inflammation. For example, release of astrocyte-derived TIMP-1 in an experimental autoimmune encephalomyelitis (EAE) model of multiple sclerosis has a protective effect and results in myelin sparing (Crocker et al., 2006) and attenuates the effect of IL-1 $\beta$ on the wound healing responses in vitro (Johnson and Crocker, 2015). The response of microglia to inflammatory stimulation and TIMP-1 appears to be more complex. Microglia play an important part of driving the initial inflammatory response in the CNS (during the "activated" M1 state), but also contribute to the resolution of inflammation (as M2) cells (Cherry et al., 2014; Popiolek-Barczyk et al., 2015). While in the M2 state, microglia can aid in inflammation resolution both through the release of IL-10 and TIMP-1 (Cherry et al., 2014; PopiolekBarczyk et al., 2015). Moreover, administration of drugs with anti-inflammatory properties shift microglia from a M1 into a M2 state, resulting in the release of TIMP-1 (PopiolekBarczyk et al., 2015). Interestingly, TIMP-1 delivery prevents the release of TNF- $\alpha$ from microglia (Nuttall et al., 2007), further supporting the anti-inflammatory, and potentially the antinociceptive, capacity of TIMP-1. Collectively, these data suggest that TIMP-1 may aid in the resolution of inflammation in peripheral and central tissues, and that TIMP-1 may be a critical component of a signaling cascade involved in reducing inflammatory hypersensitivity.

The function of TIMP-1 is also determined by the specific interactions TIMP-1 has with its binding partners, which includes both proteases and membrane-bound receptors. We have yet to identify which receptor is responsible for the antinociceptive properties of TIMP-1, but it is known that TIMP- 1 binds and activates the CD63/ $\beta 1$ integrin receptor complex (Jung et al., 2006; Toricelli et al., 2013; Takawale et al., 2017; Nicaise et al., 2019). Interestingly, prior work has shown that interfering with the interaction between $\beta 1$ integrin and versican attenuates inflammatory and neuropathic pain, as well as nociceptor activity (Dina et al., 2004; Ferrari and Levine, 2016). Consequently, keratinocyte-derived TIMP1 may bind to $C D 63 / \beta 1$ integrin expressed on cutaneous nerve endings ultimately, attenuating primary afferent function. While primary afferents are known to express $\beta 1$ integrin, it is unclear whether they also express CD63. If neurons do not express CD63 there may be an indirect pathway involving other cell types, such as mast cells or keratinocytes, where TIMP-1 may bind to CD63 to alter the release pronociceptive molecules that influence neuronal function (Bertaux et al., 1991; Baumbauer et al., 2015; Moehring et al., 2018). Interestingly, disrupting the activity of $\beta 1$ integrin prevents persistent pain in a model of hyperalgesic priming while leaving the acute phase of sensitivity intact (Dina et al., 2004). Therefore, stimulating TIMP-1 release, or the direct delivery of TIMP-1, may prevent the emergence of pathological pain, through $\beta 1$ integrin activation, while leaving the capacity to detect normally painful stimuli unaffected.

Finally, demonstrating that TIMP-1 alleviates ongoing pain in WT mice we show that TIMP-1 is a potential clinical target for therapeutic intervention. It may be possible that TIMP-1 acts as a physiological "brake" on the nociceptive 
system to prevent overexcitation of primary afferents and the development of centralized pain states. Consequently, targeting TIMP-1 may have therapeutic benefit for both peripheral and central pain. For example, while we did not directly assess hypersensitivity at somatic regions beyond the contralateral hindpaw, our data may have implications for understanding the underlying mechanisms of widespread pain syndromes, such as fibromyalgia. Our data may also have implications for understanding metastatic processes in non-painful forms of cancer. Indeed, TIMP-1 has been studied extensively in cancer (Gong et al., 2013; Toricelli et al., 2013; Jackson et al., 2017) and is therefore of significant interest for determining how metastases develop without producing pain. It is not yet clear whether painful and non-painful cancers differentially express TIMP-1 or whether TIMP-1 receptor binding kinetics are altered in painful and non-painful cancers. Understanding these dynamics may have clinical implications for developing early cancer detection strategies, especially if TIMP-1 is considered a regulator of pain state and not just one involved in tissue remodeling. Finally, if TIMP-1 can be utilized as a target for attenuating pathological pain, TIMP-1 may serve as an alternative to opioid-based medicines. This possibility is intriguing given our data showing that peripheral administration of TIMP1 has antinociceptive properties and prevents the spread of sensitivity to uninflamed somatic regions. However, these conclusions should be taken with some caution as research has shown that excess TIMP-1 expression, specifically through genetic overexpression, may lead to unintended adverse events (Grünwald et al., 2018). Therefore, future research should focus on illuminating the mechanisms by which TIMP-1 attenuates pain and hypersensitivity and how targeting this system may be therapeutically beneficial.

\section{CONCLUSION}

The major goal of this study was to investigate the role TIMP-1 may play in pathological pain states associated with inflammation and in the absence of frank tissue damage. We found TIMP-1 expression was associated with behavioral hyposensitivity immediately following inflammation, and that mice lacking TIMP-1 developed exacerbated hypersensitivity that could be prevented by rmTIMP-1 protein constructs that either inhibit MMP activity or activate membrane bound receptors. Thus, endogenous TIMP-1 may prevent the induction of pain by both, regulating MMP activity and potentially through a novel cell-receptor signaling cascade mediated by CD63. Given the dual nature of TIMP-1 activity, it may be possible to target these pathways as an innovative strategy for attenuating persistent/chronic pain.

\section{DATA AVAILABILITY}

The datasets generated for this study are available on request to the corresponding author.

\section{ETHICS STATEMENT}

The animal study was reviewed and approved by University of Connecticut Health Center Institutional Animal Care and Use Committee.

\section{AUTHOR CONTRIBUTIONS}

BK: experimental design, data collection and analysis, mouse breeding and care, manuscript preparation. NK: data collection. JH: collection of conditioned place preference data. TK: experimental design and analysis of data from conditioned place preference experiments, manuscript preparation. SC: generation of T1KO mouse line, experimental design, manuscript preparation. EY: experimental design, data analysis, manuscript preparation. KB: experimental design, data analysis, manuscript preparation, experimental oversight.

\section{FUNDING}

This research was supported by the National MS Society grant (RG-1802-30211) to SC. This research was also supported by the grants 1R03NS096454-01 and R21NS104789-01A1, and funding from the Rita Allen Foundation $(\mathrm{KB})$.

\section{ACKNOWLEDGMENTS}

We would like to thank Ms. Nicole Glidden and Ms. Jessica Yasko for their technical assistance. This manuscript has been released as a Pre-Print at bioRxiv (doi: 10.1101/540724).

\section{SUPPLEMENTARY MATERIAL}

The Supplementary Material for this article can be found online at: https://www.frontiersin.org/articles/10.3389/fnmol. 2019.00220/full\#supplementary-material

FIGURE S1 | Subcutaneous injection of saline vehicle causes mechanical hypersensitivity in mice lacking TIMP-1. Assessment of mechanical hypersensitivity over 7 days following s.c. administration of $0.9 \%$ saline. T1KO mice show a significant reduction in mechanical thresholds relative to all other mice 1 day following injection, and saline treated WT mice 5- and 7-days following injection ( $n=6 /$ condition). ${ }^{*}$ indicates significant differences compared to naïve controls, and \# indicates significant difference compared to WT mice given saline injections, $p<0.05$, and error bars depict SEM.

FIGURE S2 | Dose-response curve for rmTIMP-1 administration. Mice were administered 1,10 , or $100 \mathrm{ng} / \mu \mathrm{L}(10 \mu \mathrm{L}$ vol) s.c. at the time of CFA injection. Administration of $10 \mu \mathrm{L}$ of $r m$ TIMP-1 resulted in the greatest attenuation of mechanical hypersensitivity relative to all other doses. *indicates significantly different response thresholds relative to all other mice, and \#indicates significantly different response thresholds relative to CFA treated mice, $n=6 /$ condition, $p<0.05$, and error bars depict SEM. 


\section{REFERENCES}

Ahmed, M., King, K., Pearce, S., Ramsey, M., Miranpuri, G., and Resnick, D. (2011). Novel targets for spinal cord injury related neuropathic pain. Ann. Neurosci. 18, 162-167. doi: 10.5214/ans.0972.7531.1118413

Allchorne, A. J., Broom, D. C., and Woolf, C. J. (2005). Detection of cold pain, cold allodynia and cold hyperalgesia in freely behaving rats. Mol. Pain 1, 36-36.

Andreev, N. Y., Dimitrieva, N., Koltzenburg, M., and Mcmahon, S. B. (1995). Peripheral administration of nerve growth factor in the adult rat produces a thermal hyperalgesia that requires the presence of sympathetic post-ganglionic neurones. Pain 63, 109-115.

Baker, A., Edwards, D., and Murphy, G. (2002). Metalloproteinase inhibitors: biological actions and therapeutic opportunities. J. Cell Sci. 115, 3719-3727.

Baumbauer, K., Deberry, J., Adelman, P., Miller, R., Hachisuka, J., Lee, K., et al. (2015). Keratinocytes can modulate and directly initiate nociceptive responses. eLife 4:e09674. doi: 10.7554/eLife.09674

Bennett, G., Al-Rashed, S., Hoult, J. R. S., and Brain, S. D. (1998). Nerve growth factor induced hyperalgesia in the rat hind paw is dependent on circulating neutrophils. Pain 77, 315-322.

Bertaux, B., Hornebeck, W., Eisen, A. Z., and Dubertret, L. (1991). Growth stimulation of human keratinocytes by tissue inhibitor of metalloproteinases. J. Investig. Dermatol. 97, 679-685.

Brew, K., and Nagase, H. (2010). The tissue inhibitors of metalloproteinases (TIMPs): an ancient family with structural and functional diversity. Biochim. Biophys. Acta 1803, 55-71. doi: 10.1016/j.bbamcr.2010.01.003

Cathcart, J., and Cao, J. (2015). MMP inhibitors: past, present and future. Front. Biosci. 20:1164-1178.

Chapman, R., and Vierck, C. (2017). The transition of acute postoperative pain to chronic pain: an integrative overview of research on mechanisms. J. Pain 18, 359.e1-359.e38. doi: 10.1016/j.jpain.2016.11.004

Cherry, J. D., Olschowka, J. A., and O’banion, M. K. (2014). Neuroinflammation and M2 microglia: the good, the bad, and the inflamed. J. Neuroinflamm. 11, 98-98. doi: 10.1186/1742-2094-11-98

Claycomb, K., Johnson, K., Winokur, P., Sacino, A., and Crocker, S. (2013). Astrocyte regulation of CNS inflammation and remyelination. Brain Sci. 3, 1109-1127. doi: 10.3390/brainsci3031109

Couper, K. N., Blount, D. G., and Riley, E. M. (2008). IL-10: the master regulator of immunity to infection. J. Immunol. 180, 5771-5777.

Crocker, S. J., Whitmire, J. K., Frausto, R. F., Chertboonmuang, P., Soloway, P. D., Whitton, J. L., et al. (2006). Persistent macrophage/microglial activation and myelin disruption after experimental autoimmune encephalomyelitis in tissue inhibitor of metalloproteinase-1-deficient mice. Am. J. Pathol. 169, 2104-2116.

Cui, X., Liu, K., Xu, D., Zhang, Y., He, X., Liu, H., et al. (2018). Mast cell deficiency attenuates acupuncture analgesia for mechanical pain using c-kit gene mutant rats. J. Pain Res. 11, 483-495. doi: 10.2147/JPR.S15 2015

Dhar, A., Gardner, J., Borgmann, K., Wu, L., and Ghorpade, A. (2006). Novel role of TGF-beta in differential astrocyte-TIMP-1 regulation: implications for HIV-1-dementia and neuroinflammation. J. Neurosci. Res. 83, 1271-1280.

Dina, O. A., Parada, C. A., Yeh, J., Chen, X., Mccarter, G. C., and Levine, J. D. (2004). Integrin signaling in inflammatory and neuropathic pain in the rat. Eur. J. Neurosci. 19, 634-642.

Dixon, W. (1980). Efficient analysis of experimental observations. Ann. Rev. Pharmacol. Toxicol. 20, 441-462.

Edqvist, P., Fagerberg, L., Hallström, B., Danielsson, A., Edlund, K., Uhlén, M., et al. (2015). Expression of human skin-specific genes defined by transcriptomics and antibody-based profiling. J. Histochem. Cytochem. 63, 129-141. doi: 10.1369/ 0022155414562646

Ellis, A., and Bennett, D. L. H. (2013). Neuroinflammation and the generation of neuropathic pain. Br. J. Anaesthesia 111, 26-37. doi: 10.1093/bja/ aet 128

Fagerberg, L., Jonasson, K., Von Heijne, G., Uhlen, M., and Berglund, L. (2010). Prediction of the human membrane proteome. Proteomics 10, 1141-1149. doi: $10.1002 /$ pmic. 200900258

Ferrari, L., and Levine, J. (2016). Plasma membrane mechanisms in a preclinical rat model of chronic pain. J. Pain 16, 60-66. doi: 10.1016/j.jpain.2014.10.007

Galli, S. J., and Tsai, M. (2012). IgE and mast cells in allergic disease. Nat. Med. 18, 693-704. doi: $10.1038 / \mathrm{nm} .2755$
Gardner, J., and Ghorpade, A. (2003). Tissue inhibitor of metalloproteinase (TIMP)-1: the TIMPed balance of matrix metalloproteinases in the central nervous system. J. Neurosci. Res. 74, 801-806.

Giesler, G., and Liebeskind, J. (1976). Inhibition of visceral pain by electrical stimulation of the periaqueductal gray matter. Pain 2, 43-48.

Gomis-Rüth, F., Maskos, K., Betz, M., Bergner, A., Huber, R., Suzuki, K., et al. (1997). Mechanism of inhibition of the human matrix metalloproteinase stromelysin-1 by TIMP-1. Nature 389, 77-81.

Gong, Y., Scott, E., Lu, R., Xu, Y., Oh, W. K., and Yu, Q. (2013). Timp-1 promotes accumulation of cancer associated fibroblasts and cancer progression. PLoS One 8:e77366. doi: 10.1371/journal.pone.0077366

Grünwald, B., Schoeps, B., and KrüGer, A. (2018). Recognizing the molecular multifunctionality and interactome of timp-1. Trends Cell Biol. 29, 6-19. doi: 10.1016/j.tcb.2018.08.006

Hachisuka, J., Baumbauer, K., Omori, Y., Snyder, L., Koerber, H., and Ross, S. (2016). Semi-intact ex vivo approach to investigate spinal somatosensory circuits. eLife 5:e22866. doi: 10.7554/eLife.22866

Hargreaves, K., Dubner, R., Brown, F., Flores, C., and Joris, J. (1988). A new and sensitive method for measuring thermal nociception in cutaneous hyperalgesia. Pain 32, 77-88.

He, Y., Tian, X., Hu, X., Porreca, F., and Wang, Z. J. (2012). Negative reinforcement reveals non-evoked ongoing pain in mice with tissue or nerve injury. J. Pain 6, 598-607. doi: 10.1016/j.jpain.2012.03.011

Huang, B., Zhao, X., Zheng, L., Zhang, L., Ni, B., and Wang, Y. (2011). Different expression of tissue inhibitor of metalloproteinase family members in rat dorsal root ganglia and their changes after peripheral nerve injury. Neuroscience 193, 421-428. doi: 10.1016/j.neuroscience.2011.07.031

Hylden, J., and Wilcox, G. (1983). Intrathecal serotonin in mice: analgesia and inhibition of a spinal action of substance P. Life Sci. 33, 789-795.

Imbe, H., and Kimura, A. (2017). Attenuation of pCREB and Egr1 expression in the insular and anteriorcingulate cortices associated with enhancement of CFAevoked mechanicalhypersensitivity after repeated forced swim stress. Brain Res. Bull. 134, 253-261. doi: 10.1016/j.brainresbull.2017.08.013

Jackson, H., Defamie, V., Waterhouse, P., and Khokha, R. (2017). TIMPs: versatile extracellular regulators in cancer. Nat. Rev. Cancer 17, 38-53. doi: 10.1038/nrc. 2016.115

Jankowski, M., Rau, K., Soneji, D., Ekmann, K., Anderson, C., Molliver, D., et al. (2012). Purinergic receptor P2Y1 regulates polymodal C-fiber thermal thresholds and sensory neuron phenotypic switching during peripheral inflammation. Pain 153, 410-419. doi: 10.1016/j.pain.2011. 10.042

Jankowski, M. P., Lawson, J. J., Mcilwrath, S. L., Rau, K. K., Anderson, C. E., Albers, K. M., et al. (2009). Sensitization of cutaneous nociceptors after nerve transection and regeneration: possible role of target-derived neurotrophic factor signaling. J. Neurosci. 29, 1636-1647. doi: 10.1523/JNEUROSCI.3474-08. 2009

Ji, R.-R., Xu, Z.-Z., Wang, X., and Lo, E. H. (2009). Matrix metalloprotease regulation of neuropathic pain. Trends Pharmacol. Sci. 30, 336-340. doi: 10. 1016/j.tips.2009.04.002

Johnson, K., and Crocker, S. (2015). TIMP-1 couples RhoK activation to IL-1. Neurosci. Lett. 609, 165-170. doi: 10.1016/j.neulet.2015.10.038

Jourquin, J., Tremblay, E., Bernard, A., Charton, G., Chaillan, F. A., Marchetti, E., et al. (2005). Tissue inhibitor of metalloproteinases-1 (TIMP-1) modulates neuronal death, axonal plasticity, and learning and memory. Eur. J. Neurosci. 22, 2569-2578.

Jung, K., Liu, X., Chirco, R., Fridman, R., and Kim, H. (2006). Identification of CD63 as a tissue inhibitor of metalloproteinase-1 interacting cell surface protein. Eur. Mol. Biol. Organ. 25, 3934-3942.

Kalesnikoff, J., and Galli, S. J. (2008). New developments in mast cell biology. Nat. Immunol. 9, 1215-1223. doi: 10.1038/ni.f.216

Kawasaki, Y., Xu, Z., Wang, X., Park, J., Zhuang, Z., Tan, P., et al. (2008). Distinct roles of matrix metalloproteases in the early- and late-phase development of neuropathic pain. Nat. Med. 14, 331-336. doi: 10.1038/nm1723

Kehlet, H., Jensen, T., and Woolf, C. (2006). Persistent postsurgical pain: risk factors and prevention. Lancet 367, 1618-1625.

Kessler, W., Kirchhoff, C., Reeh, P., and Handwerker, H. (1992). Excitation of cutaneous afferent nerve endings in vitro by a combination of inflammatory mediators and conditioning effect of substance P. Exp. Brain Res. 91, 467-476. 
Kim, Y., Remacle, A., Chernov, A., Liu, H., Shubayev, I., Lai, C., et al. (2012). The MMP-9/TIMP-1 axis controls the status of differentiation and function of myelin-forming schwann cells in nerve regeneration. PLoS One 7:e33664. doi: 10.1371/journal.pone.0033664

King, T., Vera-Portocarrero, L., Gutierrez, T., Vanderah, T., Dussor, G., Lai, J., et al. (2009). Unmasking the tonic-aversive state in neuropathic pain. Nat. Neurosci. 12, 1364-1366. doi: 10.1038/nn.2407

Koscsó, B., Csóka, B., Kókai, E., Németh, Z. H., Pacher, P., Virág, L., et al. (2013). Adenosine augments IL-10-induced STAT3 signaling in M2c macrophages. J. Leukocyte Biol. 94, 1309-1315. doi: 10.1189/jlb.0113043

Kouwenhoven, M., Ozenci, V., Gomes, A., Yarilin, D., Giedraitis, V., Press, R., et al. (2001). Multiple sclerosis: elevated expression of matrix metalloproteinases in blood monocytes. J. Autoimmun. 16, 463-470.

Laedermann, C. J., Pertin, M., Suter, M. R., and Decosterd, I. (2014). Voltage-gated sodium channel expression in mouse DRG after SNI leads to re-evaluation of projections of injured fibers. Mol. Pain 10:19. doi: 10.1186/1744-8069-10-19

Lawson, J. J., Mcilwrath, S. L., Woodbury, C. J., Davis, B. M., and Koerber, H. R. (2008). TRPV1 unlike TRPV2 is restricted to a subset of mechanically insensitive cutaneous nociceptors responding to heat. J. Pain 9, 298-308. doi: 10.1016/j.jpain.2007.12.001

Lee, M., Yoon, B., Osiewicz, K., Preston, M., Bundy, B., Van Heeckeren, A., et al. (2005). Tissue inhibitor of metalloproteinase 1 regulates resistance to infection. Infect. Immun. 73, 661-665.

Li, Y., Lu, Y., Zhao, Z., Wang, J., Li, J., Wang, W., et al. (2016). Relationships of MMP-9 and TIMP-1 proteins with chronic obstructive pulmonary disease risk: a systematic review and meta-analysis. J. Res. Med. Sci. 21:12.

Livak, K. J., and Schmittgen, T. D. (2001). Analysis of relative gene expression data using real-time quantitative PCR and the 2- $\Delta \Delta \mathrm{CT}$ method. Methods 25, 402-408.

Maleki, J., Lebel, A., Bennett, G., and Schwartzman, R. (2000). Patterns of spread in complex regional pain syndrome, type I (reflex sympathetic dystrophy). PAIN $88,259-266$.

Martinho, F., Teixeira, F., Cardoso, F., Ferreira, N., Nascimento, G., Carvalho, C., et al. (2016). Clinical investigation of matrix metalloproteinases, tissue inhibitors of matrix metalloproteinases, and matrix metalloproteinase/tissue inhibitors of matrix metalloproteinase complexes and their networks in apical periodontitis. Basic Res. Biol. 42, 1082-1088. doi: 10.1016/j.joen.2016.04.001

McIlwrath, S., Lawson, J., Anderson, C., Albers, K., and Richard Koerber, H. (2007). Overexpression of neurotrophin- 3 enhances themechanical response properties of slowly adapting type lafferents and myelinated nociceptors. Eur. J. Neurosci. 26, 1801-1812.

Melemedjian, O. K., Asiedu, M. N., Tillu, D. V., Peebles, K. A., Yan, J., Ertz, N., et al. (2010). IL-6- and NGF-induced rapid control of protein synthesis and nociceptive plasticity via convergent signaling to the eIF4F complex. J. Neurosci. 30, 15113-15123. doi: 10.1523/JNEUROSCI.3947-10.2010

Melemedjian, O. K., Tillu, D. V., Asiedu, M. N., Mandell, E. K., Moy, J. K., Blute, V. M., et al. (2013). BDNF regulates atypical PKC at spinal synapses to initiate and maintain a centralized chronic pain state. Mol. Pain 9:12. doi: 10.1186/ 1744-8069-9-12

Moehring, F., Cowie, A., Menzel, A., Weyer, A., Grzybowski, M., Arzua, T., et al. (2018). Keratinocytes mediate innocuous and noxious touch via ATP-P2X4 signaling. eLife 7:e31684. doi: 10.7554/eLife.31684

Moore, C., and Crocker, S. (2012). An alternate perspective on the roles of TIMPs and MMPs in pathology. Am. J. Pathol. 180, 12-16. doi: 10.1016/j.ajpath.2011. 09.008

Mukai, K., Tsai, M., Saito, H., and Galli, S. J. (2018). Mast cells as sources of cytokines, chemokines, and growth factors. Immunol. Rev. 282, 121-150.

Nagase, H., Meng, Q., Malinovskii, V., Huang, W., Chung, L., Bode, W., et al. (1999). Engineering of selective TIMPs. Ann. N. Y. Acad. Sci. 878, 1-11.

Nagase, H., Visse, R., and Murphy, G. (2006). Structure and function of matrix metalloproteinases and TIMPs. Cardiovasc. Res. 69, 562-573.

Nakagawa, T., Kubota, T., Kabuto, M., Sato, K., Kawano, H., Hayakawa, T., et al. (1994). Production of matrix metalloproteinases and tissue inhibitor of metalloproteinases-1 by human brain tumors. J. Neurosurg. 81, 69-77.

Nicaise, A., Johnson, K., Willis, C., Guzzo, R., and Crocker, S. (2019). TIMP-1 promotes oligodendrocyte differentiation through receptor-mediated signaling. Mol. Neurobiol. 56, 3380-3392. doi: 10.1007/s12035-018-1310-7
Nuttall, R. K., Silva, C., Hader, W., Bar-Or, A., Patel, K. D., Edwards, D. R., et al. (2007). Metalloproteinases are enriched in microglia compared with leukocytes and they regulate cytokine levels in activated microglia. Glia 55, 516-526.

Okun, A., DeFelice, M., Eyde, N., Ren, J., Mercado, R., King, T., et al. (2011). Transient inflammation-induced ongoing pain is driven by TRPV1 sensitive afferents. Mol. Pain 7, 1-11. doi: 10.1186/1744-8069-7-4

Pagenstecher, A., Stalder, A. K., Kincaid, C. L., Shapiro, S. D., and Campbell, I. L. (1998). Differential expression of matrix metalloproteinase and tissue inhibitor of matrix metalloproteinase genes in the mouse central nervous system in normal and inflammatory states. Am. J. Pathol. 152, 729-741.

Parkitna, J., Korostynski, M., Kaminska-Chowaniec, D., Obara, I., Mika, J., Przewlocka, B., et al. (2006). Comparison of gene expression profiles in neuropathic and inflammatory pain. J. Pharmacol. Physiol. 57, 401-414.

Popiolek-Barczyk, K., Kolosowska, N., Piotrowska, A., Makuch, W., Rojewska, E., Jurga, A. M., et al. (2015). Parthenolide relieves pain and promotes M2 microglia/macrophage polarization in rat model of neuropathy. Neural Plast. 2015:676473. doi: 10.1155/2015/676473

Remacle, A., Hullugundi, S., Dolkas, J., Angert, M., Chernov, A., Strongin, A., et al. (2018). Acute- and late-phase matrix metalloproteinase (MMP)-9 activity is comparable in female and male rats after peripheral nerve injury. J. Neuroinflamm. 15:89. doi: 10.1186/s12974-018-1123-7

Ries, C. (2014). Cytokine functions of TIMP-1. Cell. Mol. Life Sci. 71, 659-672. doi: 10.1007/s00018-013-1457-3

Shir, Y., and Seltzer, Z. (1991). Effects of sympathectomy in a model of causalgiform pain produced by partial sciatic nerve injury in rats. PAIN 45, 309-320.

Shubayev, V. I., and Strongin, A. Y. (2018). Tissue inhibitors of metalloproteases strike a nerve. Neural Regen. Res. 13, 1890-1892.

Steinbrocker, O., Isenberg, S., Silver, M., Neustadt, D., Kuhn, P., and Schittone, M. (1953). Observations on pain produced by injection of hypertonic saline into muscles and other supportive tissues. J. Clin. Investig. 32, 1045-1051.

Stetler-Stevenson, W. G. (2008). Tissue inhibitors of metalloproteinases in cell signaling: metalloproteinase-independent biological activities. Sci. Signal 1:re6. doi: 10.1126/scisignal.127re6

Sutaeg Hwang, A., and Wilcox, G. L. (1986). Intradermal hypertonic saline-induced behavior as a nociceptive test in mice. Life Sci. 38, 2389-2396.

Tabachnick, B., and Fidell, L. (2007). Using Multivariate Statistics. Boston, MA: Allyn \& Bacon/Pearson Education.

Takawale, A., Zhang, P., Patel, V., Wang, X., Oudit, G., and Kassiri, Z. (2017) Tissue inhibitor of matrix metalloproteinase-1 promotes myocardial fibrosis by mediating CD63-integrin $\beta 1$ interaction. Hypertension 71, 1092-1103. doi: 10.1161/HYPERTENSIONAHA.117.09045

Tal, M. (1999). A role for inflammation in chronic pain. Curr. Rev. Pain 3, 440-446. Thalakoti, S., Patil, V. V., Damodaram, S., Vause, C. V., Langford, L. E., Freeman, S. E., et al. (2007). Neuron-glia signaling in trigeminal ganglion: implications for migraine pathology. Headache 47, 1008-1025.

Thevenard, J., Verzeaux, L., Devy, J., Etique, N., Jeanne, A., Schneider, C., et al. (2014). Low-density lipoprotein receptor-related protein-1 mediates endocytic clearance of tissue inhibitor of metalloproteinases- 1 and promotes its cytokinelike activities. PLoS One 9:e103839. doi: 10.1371/journal.pone.0103839

Thorne, M., Moore, C., and Robertson, G. (2009). Lack of TIMP-1 increases severity of experimental autoimmune encephalomyelitis: effects of darbepoetin alfa on TIMP-1 null and wild-type mice. J. Neuroimmunol. 211, 92-100. doi: 10.1016/j.jneuroim.2009.04.003

Toricelli, M., Melo, F., Peres, G., Silva, D., and Jasiulionis, M. (2013). Timp1 interacts with beta-1 integrin and CD63 along melanoma genesis and confers anoikis resistance by activating PI3-K signaling pathway independently of Akt phosphorylation. Mol. Cancer 12:22. doi: 10.1186/1476-4598-12-22

Tracey, D., and Walker, J. (1995). Pain due to nerve damage: are inflammatory mediators involved. Inflamm. Res. 44, 407-411.

Treede, R., Meyer, R., Raja, S., and Campbell, J. (1992). Peripheral and central mechanisms of cutaneous hyperalgesia. Prog. Neurobiol. 38, 397-421.

Treede, R., Rief, W., Barke, A., Aziz, Q., Bennett, M., Benoliel, R., et al. (2015). A classification of chronic pain for ICD-11. Pain 156, 1003-1007.

Vause, C. V., and Durham, P. L. (2010). Calcitonin gene-related peptide differentially regulates gene and protein expression in trigeminal glia cells: findings from array analysis. Neurosci. Lett. 473, 163-167. doi: 10.1016/j.neulet. 2010.01.074 
Vrontou, S., Wong, A. M., Rau, K. K., Koerber, H. R., and Anderson, D. J. (2013). Genetic identification of C fibres that detect massage-like stroking of hairy skin in vivo. Nature 493, 669-673. doi: 10.1038/nature 11810

Welser-Alves, J., Crocker, S., and Milner, R. (2011). A dual role for microglia in promoting tissue inhibitor of metalloproteinase (TIMP) expression in glial cells in response to neuroinflammatory stimuli. J. Neuroinflamm. 8:61. doi: 10.1186/1742-2094-8-61

Woolf, C. J., Safieh-Garabedian, B., Ma, Q. P., Crilly, P., and Winter, J. (1994). Nerve growth factor contributes to the generation of inflammatory sensory hypersensitivity. Neuroscience 62, 327-331.

Yang, E., Bane, C., Maccallum, R., Kiecolt-Glaser, J., Malarkey, M., and Glaser, R. (2002). Stress-related modulation of matrix metalloproteinase expression. J. Neuroimmunol. 133, 144-150.

Yokose, U., Hachiya, A., Sriwiriyanont, P., Fujimura, T., Visscher, M., Kitzmiller, W., et al. (2012). The endogenous protease inhibitor TIMP-1 mediates protection and recovery from cutaneous photodamage. J. Investig. Dermatol. 132, 2800-2809. doi: 10.1038/jid. 2012.204
Zhao, J., Seereeram, A., Nassar, M. A., Levato, A., Pezet, S., Hathaway, G., et al. (2006). Nociceptor-derived brain-derived neurotrophic factor regulates acute and inflammatory but not neuropathic pain. Mol. Cell. Neurosci. 31, 539-548.

Zimmermann, K., Hein, A., Hager, U., Kaczmarek, J. S., Turnquist, B. P., Clapham, D. E., et al. (2009). Phenotyping sensory nerve endings in vitro in the mouse. Nat. Protoc. 4, 174-196.

Conflict of Interest Statement: The authors declare that the research was conducted in the absence of any commercial or financial relationships that could be construed as a potential conflict of interest.

Copyright (C) 2019 Knight, Kozlowski, Havelin, King, Crocker, Young and Baumbauer. This is an open-access article distributed under the terms of the Creative Commons Attribution License (CC BY). The use, distribution or reproduction in other forums is permitted, provided the original author(s) and the copyright owner(s) are credited and that the original publication in this journal is cited, in accordance with accepted academic practice. No use, distribution or reproduction is permitted which does not comply with these terms. 